\title{
Transcription regulation by histone methylation: interplay between different covalent modifications of the core histone tails
}

\author{
Yi Zhang ${ }^{1,3}$ and Danny Reinberg ${ }^{2,4}$ \\ ${ }^{1}$ Department of Biochemistry and Biophysics, Curriculum in Genetics and Molecular Biology, Lineberger Comprehensive \\ Cancer Center, University of North Carolina at Chapel Hill, North Carolina 27599-7295, USA; ${ }^{2}$ Howard Hughes Medical \\ Institute, Division of Nucleic Acids Enzymology, Department of Biochemistry, University of Medicine and Dentistry of \\ New Jersey, Robert Wood Johnson Medical School, Piscataway, New Jersey 08854, USA
}

In this review, we discuss recent advances made on histone methylation and its diverse functions in regulating gene expression. Methylation of histone polypeptides might be static and might mark a gene to be or not be transcribed. However, the decision to methylate or not methylate a specific residue in the histone polypeptides is an active process that requires coordination among different covalent modifications occurring at the amino termini of the histone polypeptides, the histone tails. Below, we summarize recent advances on histone methyltransferases, and we discuss histone methylation within the context of other histone tail modifications.

\section{Histone modifications and the histone code hypothesis}

In eukaryotic cells, genes are complexed with core histones and other chromosomal proteins in the form of chromatin. The basic repeating unit of chromatin, the nucleosome, includes two copies of each of the four core histones $\mathrm{H} 2 \mathrm{~A}, \mathrm{H} 2 \mathrm{~B}, \mathrm{H} 3$, and $\mathrm{H} 4$ wrapped by $146 \mathrm{bp}$ of DNA. With the aid of additional proteins, including histone $\mathrm{H1}$, the nucleosomes are further packaged into 30$\mathrm{nm}$ fibers with six nucleosomes per turn in a spiral or solenoid arrangement (Kornberg and Lorch 1999; Hayes and Hansen 2001). The 30-nm fiber unfolds to generate a template for transcription, an 11-nm fiber or beads on a string, by a mechanism that is not entirely clear. However, it is thought that unfolding involves post-translational modifications, particularly acetylation, of the core histone amino-terminal tails.

The 11-nm fiber is also repressive to processes requiring access of proteins to DNA. Recent studies have re-

Corresponding authors.

${ }^{3}$ E-MAIL yi_zhang@med.unc.edu; FAX (919) 966-9673.

${ }^{4}$ E-MAIL reinbedf@umdnj.edu; FAX (732) 235-5294.

Article and publication are at http://www.genesdev.org/cgi/doi/10.1101/ gad.927301. vealed that there are different types of protein complexes capable of altering the chromatin, and these may act in a physiological context to modulate DNA accessibility. One family includes multiprotein complexes that utilize the energy derived from ATP hydrolysis to mobilize or alter the structure of nucleosomes (Kingston and Narlikar 1999; Vignali et al. 2000). The other family includes protein complexes that modify the histone polypeptides covalently, primarily within residues located at the histone tails (Wu and Grunstein 2000).

As an important component of the nucleosome, each core histone is composed of a structured, three-helix domain called the histone fold and two unstructured tails. Although the histone tails are dispensable for the formation of the nucleosome, they are required for nucleosome-nucleosome interaction (Luger et al. 1997) and for establishing transcriptionally repressive chromatin, referred to as heterochromatin. Transcriptionally active chromatin within the nucleus is referred to as euchromatin (Grunstein et al. 1995).

The core histone tails are susceptible to a variety of covalent modifications, including acetylation, phosphorylation, methylation, and ubiquitination (Fig. 1). Although these modifications have been known for many years, their functions are just beginning to be revealed. The identification of the first nuclear histone acetyltransferase (HAT) as a homolog of the yeast transcriptional coactivator Gcn5p (Brownell et al. 1996) fit well with an earlier observation that acetylated histones associate with transcriptionally active genes (Hebbes et al. 1988). This important finding has led to an intensive study of the function of histone acetylation in transcriptional regulation. As a result, both biochemical and genetic evidence supports an important role of histone tail acetylation in transcriptional regulation. The important discoveries include: (1) Several transcriptional coactivators such as Gcn5, p300/CBP, PCAF, TAF250, and the p160 family of nuclear receptor coactivators contain intrinsic HAT activity (Sterner and Berger 2000; Roth et al. 
Figure 1. Sites of post-translational modifications on the histone tails. The modifications shown include acetylation (purple), methylation (red), phosphorylation (green), and ubiquitination (orange). Note that Lys 9 in the $\mathrm{H} 3$ tail can be either acetylated or methylated.

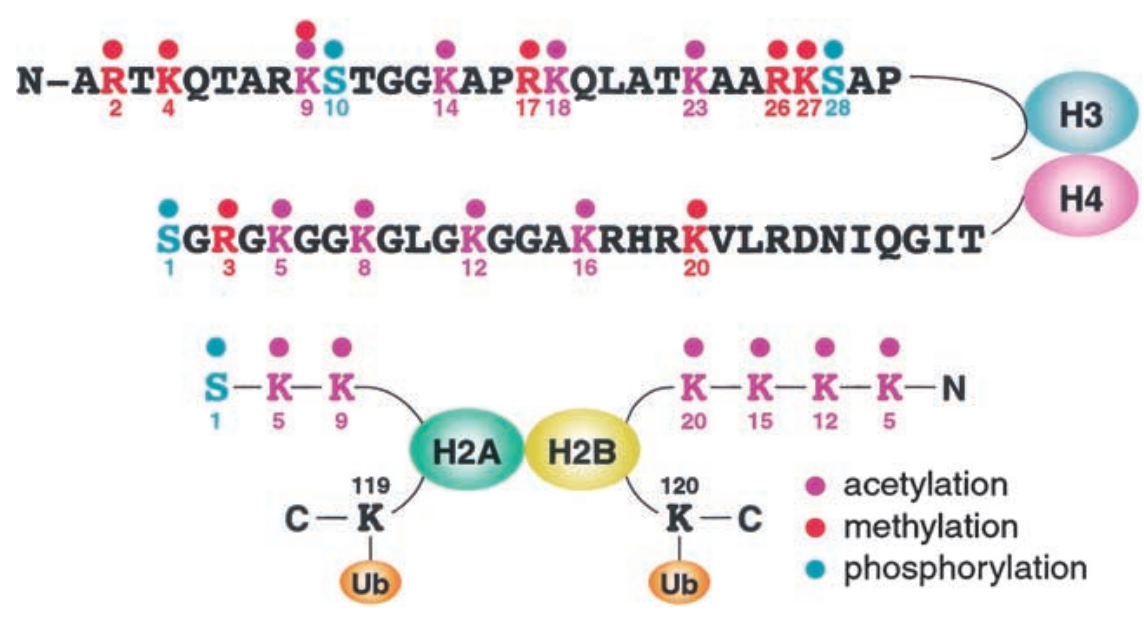

2001). (2) Global transcriptional repressors, such as Sin3 and NCoR/SMRT, among others, are associated with histone deacetylases (HDAC; Pazin and Kadonaga 1997; Kuzmichev and Reinberg 2001). (3) The enzymatic activities of HAT/HDAC are required for their transcriptional activation/repression activity (Hassig et al. 1998; Kadosh and Struhl 1998; Kuo et al. 1998; Wang et al. 1998). These studies collectively demonstrate that acetylation of histone tails regulates gene expression by affecting the dynamics of chromatin structure. In general, acetylation of core histone tails correlates with opening of chromatin structure to allow transcription.

In addition to acetylation, important progress has also been made in the studies of other types of covalent modifications including phosphorylation of histone $\mathrm{H} 3$ at Ser10 (H3-S10) and methylation of histones H3 and H4. The studies collectively reveal a complex interplay between the different covalent modifications occurring on the histone tails. These studies collectively support the histone code hypothesis (Strahl and Allis 2000). This hypothesis predicts that a pre-existing modification affects subsequent modifications on histone tails and that these modifications serve as marks for the recruitment of different proteins or protein complexes to regulate diverse chromatin functions, such as gene expression, DNA replication, and chromosome segregation. Below, we review recent progress in histone methylation and its relationship with transcriptional regulation.

\section{Protein methylation and transcriptional regulation}

Like phosphorylation, protein methylation is a covalent modification commonly occurring on carboxyl groups of glutamate, leucine, and isoprenylated cysteine, or on the side-chain nitrogen atoms of lysine, arginine, and histidine residues (Clarke 1993). Although a number of studies have indicated a role of protein methylation in signal transduction and RNA metabolism (Aletta et al. 1998; Gary and Clarke 1998), the precise function of protein methylation remains largely unknown. Because the main focus of this review is histone methyl- ation and transcriptional regulation, and histones are methylated on arginine and lysine residues only, the discussion will be limited to arginine and lysine methylation.

Arginine can be either mono- or dimethylated, with the latter in symmetric or asymmetric configurations (Fig. 2). The enzymes that catalyze this process have been divided into two types with the type I enzyme catalyzing the formation of $N^{G}$-monomethylarginine and asymmetric $N^{G}, N^{G}$-dimethylarginine residues, whereas the type II enzyme catalyzes the formation of $N^{G}$-monomethylarginine and symmetric $N^{G}, N^{\prime}$-dimethylarginine residues (Fig. 2). Similar to arginine methylation, lysine methylation on the $\varepsilon$-nitrogen can also occur as mono-, di-, or trimethylated forms (Fig. 2). Studies in the past several years have identified several RNA-associated proteins including hnRNP A1, fibrillarin, and nucleolin as substrates of type I protein arginine methyltransferase (PRMT), whereas the only substrate identified so far for type II PRMT is the myelin basic protein (Gary and Clarke 1998).

First described in 1964, histones have long been known to be substrates for methylation (Murray 1964). Early studies using metabolic labeling followed by sequencing of bulk histones have shown that several lysine residues, including lysines 4, 9, 27, and 36 of $\mathrm{H} 3$ and lysine 20 of $\mathrm{H} 4$, are preferred sites of methylation (for review, see van Holde, 1988; Strahl et al. 1999). In addition, members of the protein arginine methyltransferase family can also methylate histones in vitro /Gary and Clarke 1998). However, direct evidence linking histone methylation to gene activity was not available until recently. One major obstacle in studying the function of histone methylation is the lack of information regarding the responsible enzymes. Recent demonstration that a nuclear receptor coactivator-associated protein, CARM1 (also known as PRMT4), is a H3-specific arginine methyltransferase and that the human homolog of the Drosophila heterochromatic protein Su(var)3-9, is a H3-specific lysine methyltransferase, provided substantial evidence for the involvement of histone methylation on transcriptional regulation (Chen et al. 1999a; Rea et al. 


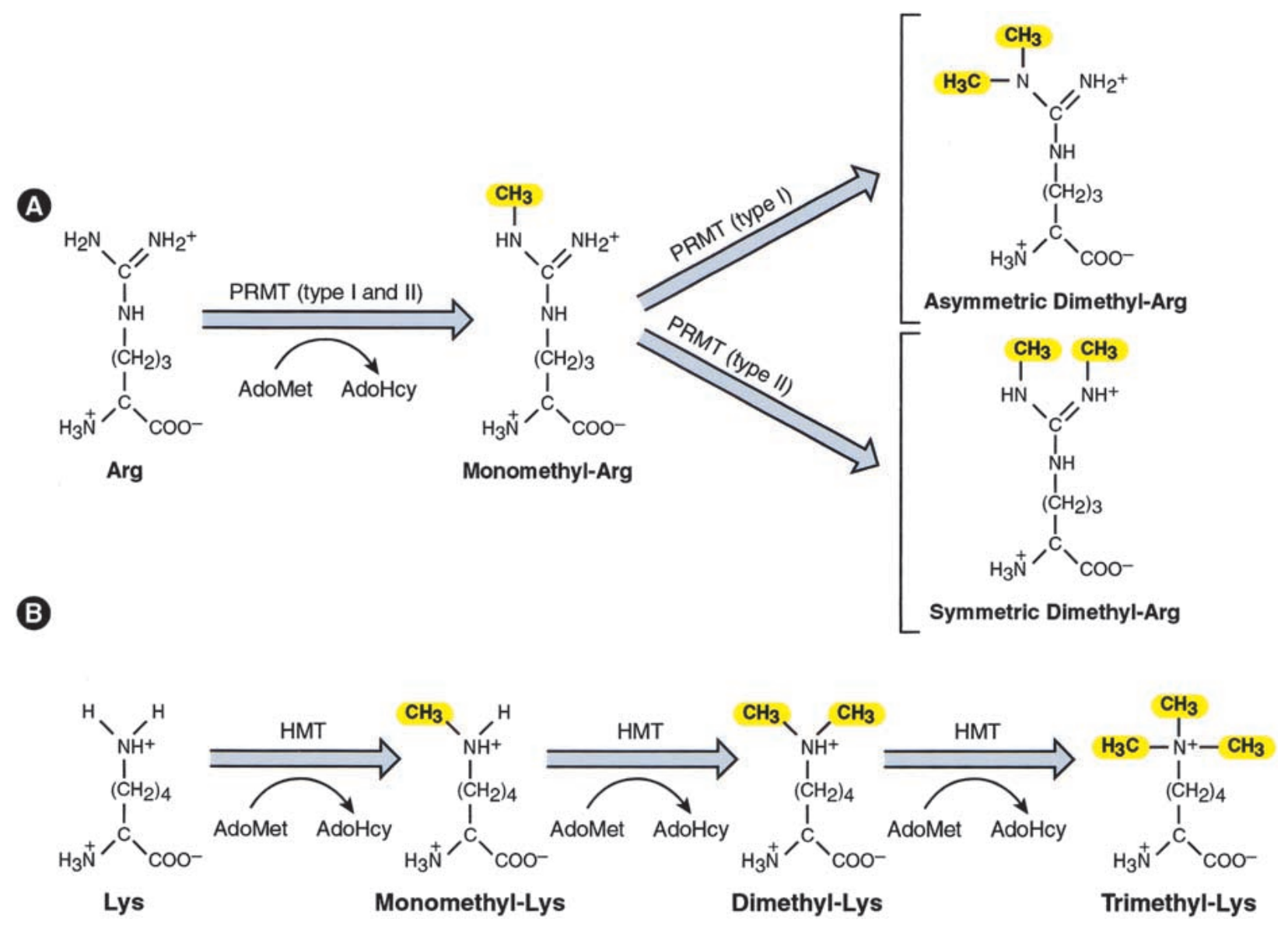

Figure 2. Chemistry of arginine and lysine methylation. $(A)$ Molecular structure of arginine, and mono- and di-methylarginine. Type I and II protein arginine methyltransferases catalyze asymmetric and symmetric dimethylation, respectively. $(B)$ Molecular structure of lysine and mono-, di-, and tri-methyl-lysine. It is not clear whether the same HMT is able to make all the different methyl-lysines.

2000). Below, we summarize recent progress in the studies of these two families of histone methyltransferases (HMTs).

\section{The PRMT family of HMTs}

Protein arginine methyltransferases (PRMTs) catalyze the transfer of methyl groups from S-adenosyl-L-methionine (SAM) to the guanidino nitrogens of arginine residues (Gary and Clarke 1998). As discussed above, PRMTs can be divided into two types on the basis of whether they catalyze symmetric or asymmetric dimethylation (Fig. 2). The recently identified H3-specific arginine methyltransferase CARM1/PRMT4 (Chen et al. 1999a), belongs to the type I protein arginine N-methyltransferase family. This family also includes PRMT1 (Lin et al. 1996) and PRMT3 (Tang et al. 1998) from mammals and RMT1/HMT1 from yeast (Gary et al. 1996; Henry and Silver 1996). These proteins share a conserved catalytic core, but have little similarity outside the core domain (Fig. 3). Sequence alignment revealed several highly conserved regions involved in SAM binding and catalysis (Fig. 3B). Therefore, it is likely that these enzymes use a similar reaction mechanism but differ with regard to substrate specificity.

PRMT1 As the founding member of the PRMT family, PRMT1 was initially identified from a yeast two-hybrid screen as a protein interacting with the immediate-early gene product TIS21 and the antiproliferative protein BTG1 (Lin et al. 1996). Recombinant PRMT1 has intrinsic protein arginine methyl-transferase activity toward arginine residues in RGG and RXR motifs of many RNAbinding proteins, including the heterogeneous nuclear ribonucleoprotein A1 (hnRNP A1). PRMT1 was also found to methylate histones in vitro (Lin et al. 1996). Indeed, a recent study designed to isolate enzymes that specifically methylate histone $\mathrm{H} 4$ resulted in the purification of PRMT1 as a major H4-specific methyltransferase (Wang et al. 2001). The PRMT1 enzymatic activity resides in a single polypeptide of $\sim 43 \mathrm{kD}$, which functions as a $350-k D$ homo-oligomer (Wang et al. 2001). In addition to interacting with BTG1 and TIS21, PRMT1 also interacts with the interferon receptor IFNAR1 and the interleukin enhancer-binding factor 3 (ILF3; Abramovich et al. 1997; Tang et al. 2000). All the PRMT1-interacting proteins appear to positively modulate the PRMT1 enzymatic activity (Lin et al. 1996; Tang 
Zhang and Reinberg

A

PRMT1 (human)

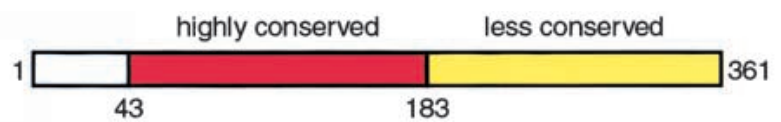

PRMT2 (human)

\begin{tabular}{|l|l|l|l|}
\hline & SH3 & & \\
\hline $33 \quad 88$ & 102 & \\
\hline
\end{tabular}

PRMT3 (human)

\begin{tabular}{|ll|l|l|}
\hline & $Z_{n^{++}}$Finger & & \\
\hline 29 & 52 & 201 & 341
\end{tabular} 512

PRMT4/CARM1

(mouse)

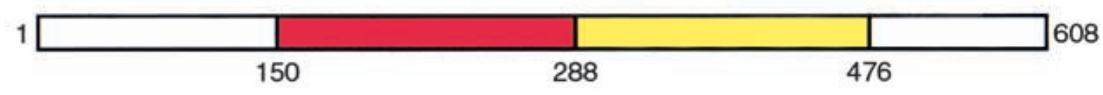

PRMT5 (human)

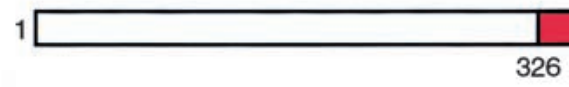

637

B

hPRMT1

hPRMT3

hPRMT2

MPRMT4

hPRMT5

Consensus

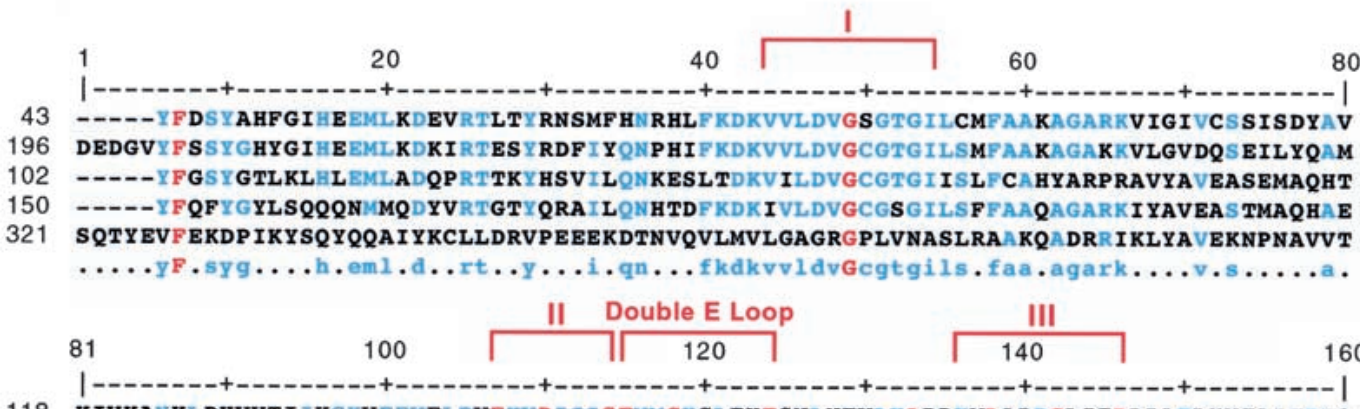

hPRMT1

118 KIVKANKLDHVVTIIKGKVEEVELPVEKVDIIISEWMGYCLFYESNLNTVLYARDKWLAPDGLIFPDRATLYVTAIEDRQ

hPRMT3

276 DIIRL N KLEDTITLIKGKI EEVHLPVEKVDVII SEWMGY FLLFES MLDSVLYAKNKYLAKGGSVYPDICTIS LVAVSDVN

hPRMT2

177

GQLVLQNGFAD I I TVYQQKVEDVVLPEKVDVLV SEWMGTCLLFEFMIES ILYARDAWLKEDGV IWP TMAALHLVPCSADK

MPRMT4

225

hPRMT5

401

VLVKS NNLTDRIVVIPGKVEEV SLP-EQVDIIISEPMGYMLFNERMLES YLHAK-KYLKPSGNMFPTIGDVHLAPFTDEQ

LENWQFEEWGSQVTVVSSDMREWVAPEKADIIVSELLGSFADNELSPECLDGAQHF-LKDDGVS I PGEYTSFLAP ISSSK

....n.1...i..i.gk.eev,lp.EkvDiiisEwmGy.1..E.mles.lya..k.Lk,dG...P...t...p.sd..

Consensus

161

180

200

220

240

hPRMT1

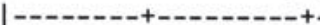

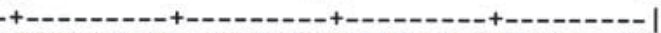

198 YKDYKIHWWENVYGFDMSCIKDVAIKEPLVDV------VDPKQLVTNACLIKEVVIYTVKVEDLTFTSPFCLQVKR---ND

356 KHADRIAF WDVYGF KM SCMKKAVIPEAVVEV-----LDP KTLISEPCG IKHIDCHTTSISDLEFSSDFTLKITR---TS

$\begin{array}{lll}\text { hPRMT2 } & 256 & \text { D YRS KVLFWDNAYEFNLSALKSLAVKEFFSKPKYNHILKPEDCLSEPCTILQLDMRTVQISDLETLRGELRFIRKAGTL } \\ \text { MPRMT4 } & 303 & \text { L YMEQFTKANFRYQPSFHGVDLSALRGAAVDEYFRQPVVDTFDIRILMAKSVKYTVNFLEAKEGDLHRIEIPFKFHMLHS }\end{array}$

hPRMT5 480 LYNEVRACREKDRDPEAQFEMPYVVRLHNFHQLSAPQPCFTFSHPNRDPMIDNNRYCTLEFPVEVN-

Consensus

..$\ldots \ldots$....

THW Loop

$241260 \quad \begin{array}{llll}280 & 300 & 320\end{array}$

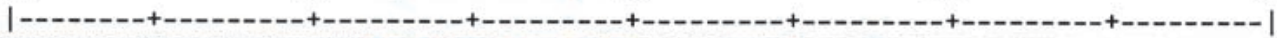

hPRMT1 270 YVHALVAYFNIEFTR-CHKRTGFSTS PESP YTHWKQTVFYMEDYLTVKTGEEIFGTIGMRPNAKNNRDLDFTIDLDFKGQ

hPRMT3 428 MCTAIAGYFDIYFEKNCHNRVVFSTGPSTKTHWKQTVFLLEKPFS VKAGEALKGKVTVHKNKKDPRSLTVTLTLNNSTQ

hPRMT2 337 HGFTAWF SVHFQSLQEGQPPQVLST TPFHP TTHWKQTLF MMDDPVPVHTGDVVT GSVVQR PVWR RMMSVALSWAVTSR

MPRMT4 383 GLVHGLAFWF DVAFIGS IMTVWLSTAP TEPLTHWYQVRCLFQS PLFAKA GDTLS TCLLIANKRQS YDIS IVAQVDQTGS

hPRMT5 546 -

Consensus

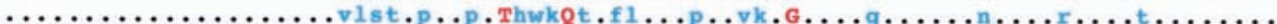

Figure 3. The PRMT protein family. (A) Schematic representation of five mammalian PRMT proteins. The conserved core region is shown in red (highly conserved) and yellow (less conserved). Numbers represent amino acids. The locations of the SH3 domain in PRMT2 and the zinc-finger domain in PRMT3 are also indicated. (B) Sequence alignment of the conserved PRMT core region. The sequences used in the alignment include human PRMT1 (Q99873), human PRMT2 (P55345), human PRMT3 (O60678), mouse PRMT4 (AF117887), and human PRMT5 (AF015913). The alignment was performed using the MultAlin program (www.toulouse.inra.fr/ multalin.html). Amino acids with $100 \%$ and $>60 \%$ conservation are shown in red and blue, respectively. The signature methyltransferase regions I, II, and III are indicated. The two loops (Double E loop and the THW loop) that form the active site (Fig. 4B) of PRMT3 are indicated. The amino acid numbers of each protein are indicated.

et al. 2000). PRMT1 has been implicated in regulating multiple cellular processes through methylating pro- teins involved in nuclear-cytoplasm transport, signal transduction, and transcription. 
Nuclear-cytoplasm transport: Although PRMT1 can methylate several proteins in vitro /Gary and Clarke 1998), the in vivo substrates for PRMT1 are largely unknown. However, studies on the potential yeast PRMT1 homolog ODP1/RMT1/HMT1 have indicated that Np13p, an abundant yeast hnRNP implicated in prerRNA processing and RNA transport (Russell and Tollervey 1992; M.S. Lee et al. 1996), is likely to be one of its substrates in vivo. ODP1, the gene encoding yeast PRMT, was originally identified as an open reading frame downstream of the gene PDX3 (Loubbardi et al. 1995). A subsequent study demonstrated that ODP1 encodes a predominant protein-arginine methyltransferase activity in yeast and was named RMT1 (Gary et al. 1996). Interestingly, this same gene was also identified in a genetic screen aimed at isolating proteins that interact with $\mathrm{Np} 13 \mathrm{p}$ (Henry and Silver 1996). Because the gene product is able to methylate the hnRNP protein Np13p in vitro and in vivo, it was named HMT1 (Henry and Silver 1996). Although RMT1/HMT1 is not essential for normal cell growth, cells lacking RMT1/HMT1 and also bearing mutations in the mRNA-binding protein $\mathrm{Np13p}$ are not viable. Mutations in the SAM-binding site of PRMT1 were shown to block methylation of Np13p in vivo, providing evidence that $\mathrm{Np} 13 \mathrm{p}$ is an in vivo substrate (McBride et al. 2000). Importantly, these mutations affected the nuclear-cytoplasm transport of Np13p. In addition, PRMT1-mediated methylation of another hnRNP, Hrp1p, was also shown to facilitate its nuclear export (Shen et al. 1998). Therefore, one function of arginine methylation in yeast is to regulate subcellular localization of proteins. Because correct subcellular localization of $\mathrm{Np} 13 \mathrm{p}$ is necessary for pre-rRNA processing and RNA transport (Russell and Tollervey 1992; M.S. Lee et al. 1996), RMT1/HMT1-mediated Np13p methylation plays an important role in RNA metabolism. Consistent with a fundamental role in RNA metabolism, homozygous PRMT1 mutant mouse embryos failed to develop beyond E6.5. However, at the cellular level, Prmt1 does not seem to be required for cell survival because PRMT1 null ES cells are viable (Pawlak et al. 2000).

PRMT1 and interferon-mediated signal transduction: In addition to regulating protein localization and early embryonic development, several lines of evidence suggest that PRMT1 regulates the interferon signaling pathway. First, a yeast two-hybrid screen revealed an interaction between PRMT1 and the IFNAR1 chain of the IFN $\alpha / \beta$ receptor. Importantly, disrupting PRMT1 expression through an antisense approach altered the antiproliferative effects of interferon (Abramovich et al. 1997). More recent studies indicate that PRMT1 regulates the transcriptional activity of STAT1 (signal transducers and activators of transcription) by methylating the protein on Arg 31, a residue conserved in all seven mammalian STATs (Mowen et al. 2001). STAT1 is a latent cytoplasmic transcription factor that is activated by a large number of extracellular signals including growth factors and cytokines (Schindler and Darnell 1995). Methylation of STAT1 by PRMT1 enhances its DNA-binding activity. Importantly, inhibition of the PRMT1 methyltransferase activity by the methylation inhibitor 5 '-methyl-thioadenosine (MTA), crippled STAT1-mediated transcriptional activation and interferon responsiveness (Mowen et al. 2001). Because MTA accumulation in transformed cells correlates with the lack of interferon responsiveness, methylation of STAT1 is likely to play an important role in the regulation of cellular proliferation (Mowen et al. 2001). Therefore, these studies collectively suggest that PRMT1-mediated STAT1 methylation regulates both STAT1 transcriptional activity and cellular proliferation.

Histone methylation and transcription activation: In addition to methylating transcription factors such as STAT1, PRMT1 also participates in transcriptional regulation through methylation of core histones. Although PRMT1 methylates histones in vitro (Lin et al. 1996), the ability of histones to serve as substrates for PRMT1 in vivo was questioned for two reasons. First, histones are much less efficient substrates of PRMT1 when compared with hnRNP A1 (Gary and Clarke 1998). Second, in vivo labeling experiments have shown that bulk histones are methylated on lysines only (Strahl et al. 1999). However, recent studies using mass spectrometry and a highly specific antibody recognizing histone $\mathrm{H} 4$ methylated at Arg 3 have unequivocally demonstrated that histone $\mathrm{H} 4$ is a substrate for PRMT1 in vivo (Wang et al. 2001; Strahl et al. 2001).

In an effort to isolate enzymes that methylate core histones, Wang and colleagues purified one of the most abundant H4-specific HMTs from HeLa cells. The enzymatic activity correlated with a single polypeptide identified as PRMT1 and the methylation site was identified to be Arg 3 (Wang et al. 2001). Importantly, an antibody that specifically recognized methylated H4-R3 recognized histone $\mathrm{H} 4$ purified from HeLa cells (Wang et al. 2001; Strahl et al. 2001). Methylation on H4-R3 is not limited to mammalian cells, as the highly specific antibody also recognized $\mathrm{H} 4$ purified from chicken and yeast (Strahl et al. 2001). PRMT1 is likely to be the most prominent methyltransferase responsible for methylation of H4-R3, as methylation on this residue was not detected when histone H4 was isolated from PRMT1null ES cells (Wang et al. 2001). Consistent with potential roles of PRMT1 in transcription, PRMT1-mediated methylation of H4-R3 facilitates subsequent acetylation of histone H4 by p300 (Wang et al. 2001). This observation provides a molecular explanation to the recent demonstration that PRMT1 function as a transcriptional coactivator of nuclear hormone receptors when coexpressed with members of the p160 family of coactivators (Koh et al. 2001). Significantly, the enzymatic activity of PRMT1 is required for its coactivator function, because a mutation of the SAM-binding site of PRMT1 crippled PRMT1 enzymatic activity as well as PRMT1 coactivator function (Wang et al. 2001). Thus, the above studies demonstrate that PRMT1 plays an important role in 
methylation of H4-R3 and its histone methyltransferase activity is involved in transcriptional activation.

CARM1/PRMT4 The coactivator-associated arginine methyltransferase 1 (CARM1) was identified in a yeast two-hybrid screen using as bait the carboxy-terminal domain of GRIP1, a member of the p160 family of coactivators (Chen et al. 1999a). Extensive sequence homology between the central portion of CARM1 and members of the PRMT family prompted the testing of CARM1 for methyltransferase activity. CARM1 preferentially methylates histone H3 in vitro (Chen et al. 1999a), and mapping of the residues demonstrated specificity for Arg 2, Arg 17, and Arg 26 (Schurter et al. 2001). CARM1 also methylates the carboxyl terminus of histone $\mathrm{H} 3$ at one or more of the four arginine (128/129/131/134) residues (Schurter et al. 2001). However, which residue(s) is/are methylated by CARM1 in vivo remains to be determined.

CARM1 is a coactivator that participates in transcriptional regulation in the presence of the p160 family of coactivators. Importantly, a mutation in the putative SAM-binding domain of CARM1 substantially reduced both its methyltransferase and coactivator activities (Chen et al. 1999a). This finding strongly suggests that arginine methylation is the molecular mechanism of transcriptional coactivation by CARM1. However, whether histones or other polypeptides are the substrate of CARM1 remains an open question. Recent studies demonstrated that the histone acetyltransferase p300 and CARM1 synergistically enhance transcription from the estrogen receptor (Koh et al. 2001). Consistent with the findings that CARM1 functions together with the p160 family of coactivators, the synergy between CARM1 and p300 requires GRIP1 (Chen et al. 2000). Of relevance in understanding the function of CARM1 in transcription is to know whether the synergistic affect between p300 and CARM depends on their enzymatic activity. Elucidating these functional interactions is likely to reveal new insights into the interplay between histone acetylation and histone methylation (see below) and is likely to shed light on coactivator activities directly regulated by acetylation and/or methylation.

Other members of the PRMT family In addition to PRMT1 and CARM1/PRMT4, three other mammalian proteins belonging to the PRMT family have been reported. The gene encoding PRMT2 was identified by screening the EST (expressed sequence tag) databases (Katsanis et al. 1997). Whether PRMT2 possesses protein arginine methyltransferase activity remains to be demonstrated.

PRMT3 was identified in a yeast two-hybrid screen using PRMT1 as bait (Tang et al. 1998). PRMT3 functions as a monomer and is predominantly localized in the cytoplasm (Tang et al. 1998). Recombinant PRMT1 and PRMT3 seem to have distinctive substrate specificity. In hypomethylated rmt1-derived extracts, GSTPRMT1 and GST-PRMT3 methylate a 45-kD protein and a $28-\mathrm{kD}$ protein, respectively. Both proteins, however, methylate a 14-kD species (Tang et al. 1998). A unique feature of PRMT3 is the presence of a zinc finger at its amino terminus (Fig. 3A). This zinc finger is required for recognition of RNA-associated substrates in RAT1 cell extracts and, therefore, appears to play an important role in determining substrate specificity of PRMT3 (Frankel and Clarke 2000).

PRMT5 is the newest member of the PRMT protein family. It was first identified as a human homolog of the Schizosaccharomyces pombe Shk1 kinase-binding protein 1, SKB1Hs (Gilbreth et al. 1998) and was later identified as a Jak (Janus kinase)-binding protein (JBP1) in a yeast two-hybrid screen using Jak2 as bait (Pollack et al. 1999). Amino acid sequence similarity between SKB1/ JBP1 and the Saccharomyces cerevisiae homolog HSL7 (histone synthetic lethal 7) and other members of the PRMT protein family suggested that this protein might be a protein arginine methyltransferase. This activity was demonstrated with recombinant JBP1 and Hs17p (Pollack et al. 1999; Lee et al. 2000; Rho et al. 2001), and the protein was renamed PRMT5. PRMT5 is localized predominantly in the cytoplasm (Rho et al. 2001) and is able to methylate myelin basic protein, fibrillamin, and histones H2A and H4 in vitro (Pollack et al. 1999; Lee et al. 2000; Rho et al. 2001). Whether any of these proteins are substrates of PRMT5 in vivo remains to be determined. Although most PRMTs belong to the type I protein arginine methyltransferase family, recent studies have revealed that PRMT5 can catalyze the formation of symmetric dimethylarginine and, therefore, belongs to type II enzyme family (Branscombe et al. 2001). The exact function of PRMT5 remains to be elucidated, but studies described below suggest that PRMT5 might be involved in mitosis.

Skb1 was found to be a negative regulator of mitosis as the protein associates with the cdc2 complex in $S$. pombe, and skbl-null mutants exhibited phenotypes characteristic of mitotic inhibitors (Gilbreth et al. 1998). Moreover, HSL7 was found to be a negative regulator of the Swe1 kinase, an important cell cycle regulator (Ma et al. 1996). Therefore, PRMT5 likely plays an important role in cell cycle regulation. Whether this function is mediated through histone polypeptides remains to be defined. It is potentially important, however, that HSL7 was initially identified in a genetic screen scoring for genes whose mutation is lethal in combination with a deletion of the histone H3 tail (Ma et al. 1996).

The above results suggest a role for PRMT5 in mitosis as well as in regulating the interferon signal transduction pathway. This later finding with PRMT5 revealed a function similar to the function of PRMT1 in regulating STAT1 (Mowen et al. 2001). Whether STAT1 can also be methylated by PRMT5 remains to be determined.

Structure of the PRMT proteins Great progress has been made recently in determining the crystal structures of two members of the PRMT family, the rat PRMT3 (Zhang et al. 2000) and the yeast Rmt1/Hmt1 (Weiss et al. 2000). The PRMT proteins vary in size but they all 
contain conserved catalytic core regions (Fig. 3). Crystallographic studies of the core region of the rat PRMT3 have revealed the following features: (1) The overall structure of the PRMT3 core can be divided into two domains-an AdoMet-binding domain and a barrel-like domain (Fig. 4A). The AdoMet-binding domain is a typical Rossmann fold (green) plus two amino-terminal helices (red) with a bound AdoHcy (S-adenosyl-homocysteine) (light blue). (2) The active site is situated in a deep pocket between the two domains. The residues that make up the active site are conserved across the PRMT family, consisting of a 12-residue double-E loop containing two invariant Glu (E326 and E355 in rat PRMT3) and one His-Asp proton-relay system (Fig. 4B). It is believed that the pocket would accommodate the side chain of an arginine so that the terminal amino group could reach the AdoMet (Zhang et al. 2000). The residues lining the pocket include the negatively charged Glu 326 and Glu 355, and the hydrophobic amino acids Phe 334 and Tyr 330. This arrangement fits well with the asymmetric polarity of the substrate arginine residues. In the absence of the target arginine, the active site is occupied by five ordered water molecules, three of which (sites 1, 2, and 3) directly interact with Glu 326, Glu 335, and His 476 (Fig. 4B). (3) PRMT3 forms a dimer mediated by a three-helix insertion into the barrel domain.

Crystallographic studies of the yeast arginine methyltransferase Rmt1/Hmt1 revealed that Hmt1 forms a hexamer composed of three dimers (Weiss et al. 2000). The surface of the oligomer is dominated by large acidic cavities at the dimer interfaces. The ability of Hmt 1 to oligomerize is critical for its enzymatic activity as mutations disrupting the dimer contact sites eliminated its enzymatic activity both in vitro and in vivo (Weiss et al. 2000). Similar to PRMT3, the Hmt1 monomer also consists of two domains (Weiss et al. 2000). These structure features suggest that all the PRMT family members are likely to have a similar core domain structure and, therefore, use a similar mechanism for catalysis. The differences are likely to reside in the nonconserved regions, that is, the amino- and/or carboxy-terminal regions, which likely determine the substrate specificity and the ability to form oligomers.

\section{SET domain family of HMTs}

The SET domain is an evolutionarily conserved sequence motif initially identified in the Drosophila position effect variegation (PEV) suppressor SU(VAR)3-9 (Tschiersch et al. 1994), the Polycomb-group protein Enhancer of zeste (Jones and Gelbart 1993), and the trithorax-group protein Trithorax (Stassen et al. 1995). Over 200 proteins of diverse functions, ranging from mammals to bacteria and viruses, have been identified to contain this motif (http://sMART.embl-heidelberg.de/smart/ do_annotation.pl?DOMAIN=SET\&BLAST=DUMMY). A major function of the SET domain-containing proteins is to modulate gene activity (Jenuwein et al. 1998). However, the underlying mechanism is not understood. A clue that the SET domain may be an important signature motif for protein methyltransferases came from studies on several plant SET-domain-containing proteins, where it was found that several of the proteins possessed protein methyltransferase activity (Klein and Houtz 1995; Zheng et al. 1998). We discuss SET domain proteins and histone methylation below.

SUV39H1 and SUV39H2 As one of the founding members of the SET domain protein family, Drosophila Su(var) 3-9 was identified in genetic screens aimed at isolating suppressors of PEV (Tschiersch et al. 1994). In ad-
A

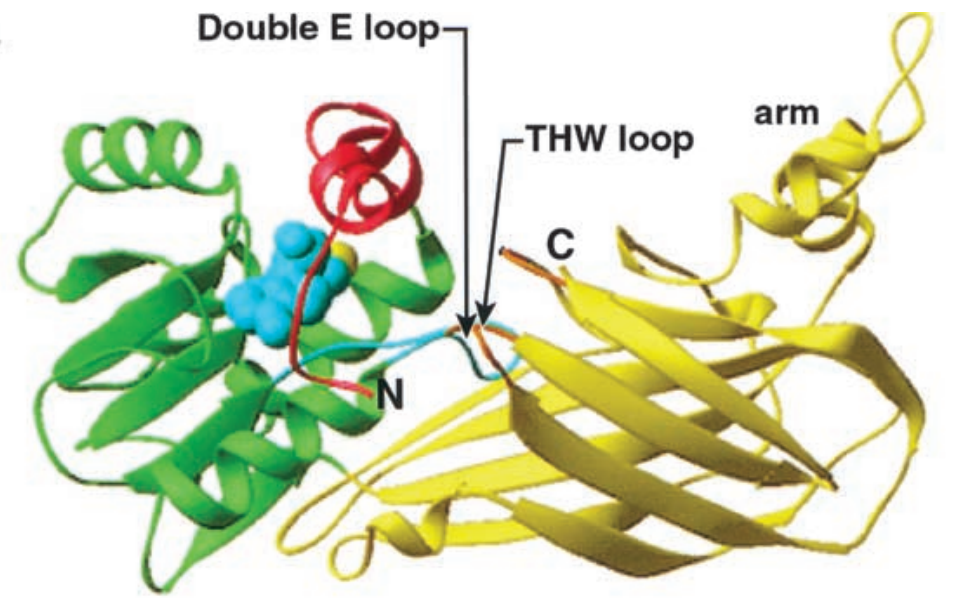

B

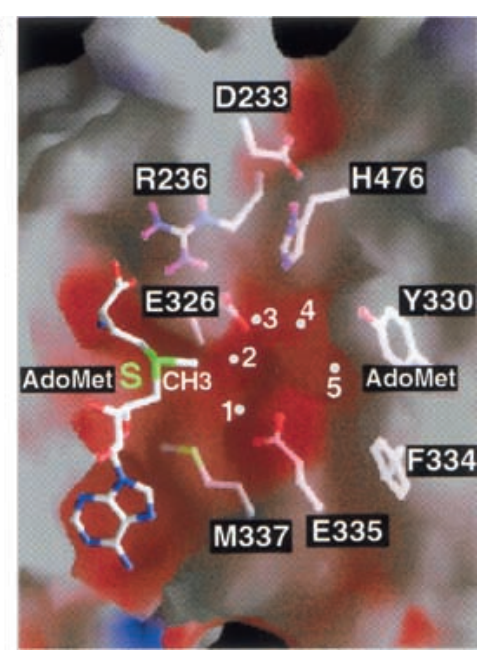

Figure 4. Structure of the rat PRMT3 catalytic core in complex with reaction product AdoHcy. (A) Overall structure of the PRMT3 core domain. The two amino-terminal helices (red), the AdoMet-binding domain (green), the barrel-like structure (yellow), and the three-helix arm (yellow) are indicated. The active site, composed of the double E loop and the THW loop, are also indicated. (B) A close view of the active site pocket. The amino acid number corresponds to the rat PRMT3. The five water molecules $(1-5)$ in the active site are indicated. Courtesy of Dr. Xiaodong Cheng. 
dition to the SET domain, the Su(var) 3-9 protein also contains an evolutionarily conserved chromodomain found in a group of chromatin-related proteins (Koonin et al. 1995). Mutations in the fission yeast homolog clr4 disrupt the association of Swi6p with heterochromatin and result in chromosome segregation defects (Ekwall et al. 1996). Studies with the human (SUV39H1), and mouse (Suv39h1) homologs of Su(var)3-9 demonstrated that the encoded polypeptide associates with the mammalian heterochromatic protein HP1, a homolog of Swi6p (Aagaard et al. 1999). Therefore, the function of SUV39H1/Clr4 and HP1/Swi6p in heterochromatic gene silencing seems to be conserved from yeast to human. Core histones have long been known to play important roles in heterochromatic gene silencing /Grunstein 1998), but the role of histone methylation in heterochromatin silencing was not known until recently. The demonstration that Suv39H1 and Clr4 possess intrinsic histone methyltransferase activity supports a role of histone methylation in heterochromatin silencing (Rea et al. 2000).

The sequence similarity between the SET domain of SUV39H1 and some plant SET-domain-containing methyltransferases (Klein and Houtz 1995; Zheng et al. 1998) prompted Jenuwein and colleagues to test for possible methyltransferase activity associated with SUV39H1. This study revealed that SUV39H1 and its S. pombe homolog Clr4 contain intrinsic histone methyltransferase activity. The enzyme specifically methylates histone $\mathrm{H} 3$ on lysine 9 (H3-K9; Rea et al. 2000). Mutagenesis studies with Suv39H1 showed that the SET domain and two adjacent cysteine-rich regions (preSET and postSET) are required for enzymatic activity (Fig. 5). Consistent with the requirement of these three regions for enzymatic activity, other SET domain-containing proteins that lack one or more of the cysteine-rich regions, for example, recombinant EZH2 and TRX, do not contain histone methyltransferase activity in vitro (Rea et al. 2000). It is important to point out, however, that it is not known whether the native EZH2 and TRX protein complexes have HMT activity. Moreover, there are other SET domain-containing proteins that are devoid of one or both of the cysteine-rich domains, yet contain histone methyltransferase activity (Fig. 5 and see below).

Although Suv39h-null primary mouse embryonic fibroblasts (PMEFs) exhibited mitotic defects, the methylation level of H3-K9 of bulk histones appears unchanged when compared with those derived from wild-type cells (Rea et al. 2000); therefore, additional H3-K9-specific methyltransferases exist in the cell. Consistent with this idea, a second murine K9-specific HMT was recently identified (O'Carroll et al. 2000). This murine gene, termed Suv39h2, encodes a protein that is $59 \%$ identical to the murine Suv39h1. The major difference between these two proteins is the presence of a highly basic amino terminus in Suv39h2. Although Suv39h1 and Suv39h2 display overlapping expression profiles during mouse embryogenesis, Suv39h2 transcripts are uniquely expressed in the testis of adult mice. Because Suv39h2 specifically accumulates on the sex chromosomes (XY body), which undergo transcriptional silencing during the first meiotic prophase, the HMT activity of Suv39h2 was proposed to be involved in organizing meiotic heterochromatin and genomic imprinting of the male germ line (O'Carroll et al. 2000). The proposed functions for Suv39h2 remains to be demonstrated.

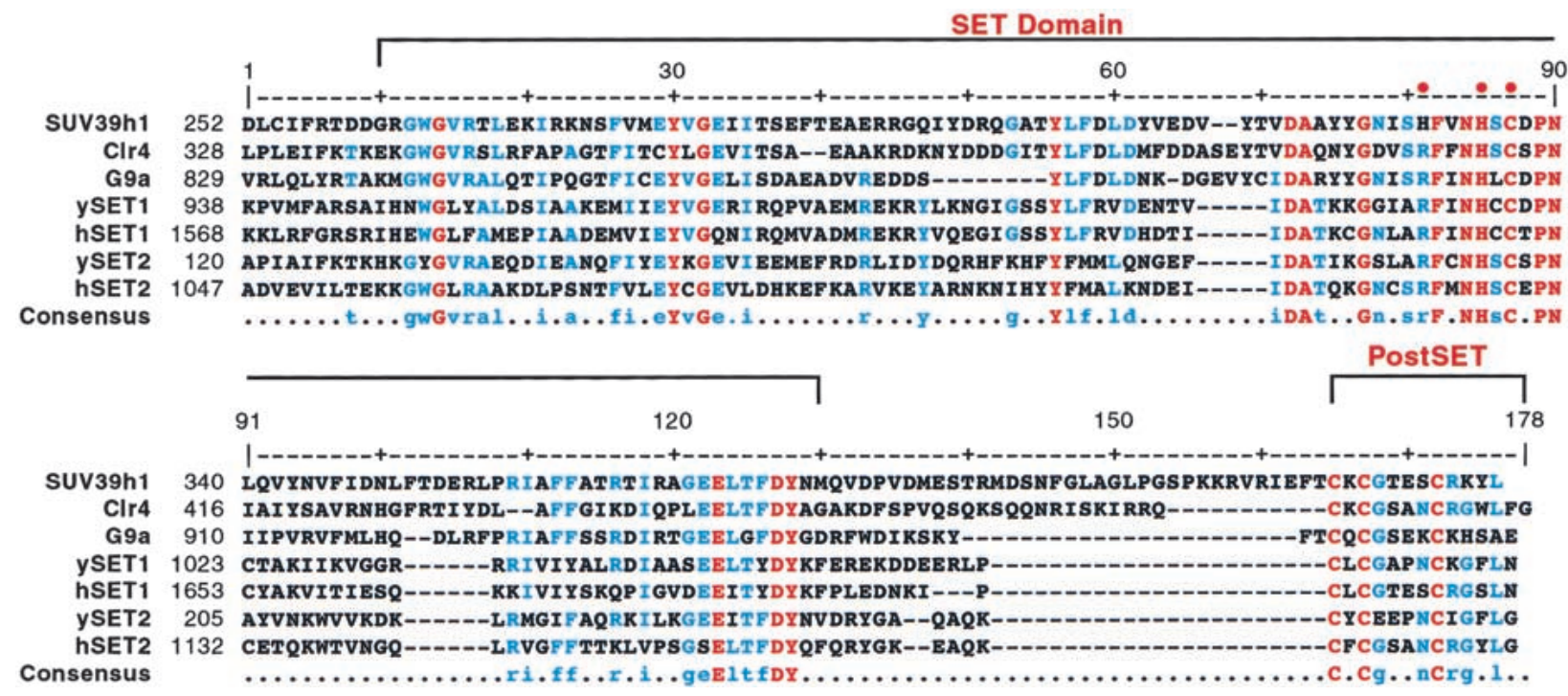

Figure 5. Sequence alignment of the SET and postSET domains of known HMTs. The sequences used in the alignment include human SUV39h1 (NP_003164), S. pombe Clr4 (T43945), human G9a (NP_006700), S. cerevisiae SET1 (P38827) and SET2 (P46995), and their potential human homologs (AB002337 and AJ238403). The alignment was performed with the MultAlin program (www.toulouse.inra.fr/multalin.html). Amino acids with $100 \%$ and $>50 \%$ conservation are shown in red and blue, respectively. SET and postSET domains are indicated. The amino acids known to affect HMT activity are indicated by red dots on top of the sequence. The amino acid numbers of each protein are also indicated. 
G9a and other SET domain-containing proteins In addition to the SUV39H1 and SUV39H2 HMTs, another SET domain-containing protein G9a has recently been shown to be an HMT (Tachibana et al. 2001). The gene encoding G9a is located in the class III region of the human major histocompatibility complex locus (Milner and Campbell 1993). Like the Suv39h family of proteins, G9a contains the SET domain as well as the adjacent cysteine-rich preSET and postSET domains (Rea et al. 2000). In addition, G9a also contains a polyglutamic acid stretch and six continuous ankyrin repeats at its amino terminus. The existence of a SET domain and adjacent cysteine-rich regions prompted Tachibana and colleagues to test the ability of G9a protein to methylate core histones. Like the Suv39h family of HMTs, G9a is able to specifically methylate histone $\mathrm{H} 3$ at lysine 9 , but also at lysine 27, a site reported to be methylated in vivo (Strahl et al. 1999). Interestingly the two H3-K residues targeted by G9a in vitro are contained within strikingly similar amino acid sequences, suggesting that G9a might recognize the lysine residue within a motif (TKXXARKS). The specific activity of G9a is 10- to 20-fold higher than that of Suv39h1 (Tachibana et al. 2001). Fluorescent labeling studies indicated that G9a and Suv39h1 have distinctive localization patterns suggesting that these enzymes perform distinct functions.

A database search revealed that, in addition to G9a and the Suv39h1 family of proteins, other proteins with SET, preSET, and postSET domains exist. For example, CLLD8, a candidate gene for leukemogenesis at chromosome 13q14, contains a split SET domain and the adjacent preSET and postSET cysteine-rich regions (Mabuchi et al. 2001). Interestingly, this protein also contains a region with similarity to the methyl-CpG-binding domain (MBD) of the MeCP2 protein (Hendrich and Bird 1998). Similar bifurcated SET, preSET, postSET, and MBD domains were also found in the SETDB1 protein (Harte et al. 1999) and its mouse homolog ESET, which interacts with ERG, a member of the ETS-family of transcription factors (Liu Yang, pers. comm.). Preliminary studies indicate that ESET possesses H3-specific HMT activity, raising the possibility that the transcription factor ERG may participate in transcriptional regulation through ESET-mediated histone methylation (Y.Z, unpubl.). The finding that a split SET domain-containing protein possesses HMT activity suggests that the SET domain can be separated into two subdomains without destroying its enzymatic activity.

Is the SET domain sufficient for enzymatic activity? The SET domain-containing HMTs described above all contain the preSET and postSET domains believed to be required for their enzymatic activity (Rea et al. 2000). Analysis of the $S$. cerevisiae genome database identified six SET domain-containing proteins, yet none of these proteins contains both the preSET and postSET domains. Indeed, only Set 1 and Set 2 proteins contain the postSET domain (Fig. 5), the other proteins only contain the SET domain. The histones in yeast are clearly methylated (Strahl et al. 1999), and the specificity of Set1 [H3-K4 (D.
Allis and T. Kouzarides, pers. comm.)] and Set2 [core domain of histone $\mathrm{H} 3$ (T. Kouzarides, pers. comm.)] proteins do not account for all the histone methylation observed in yeast. Therefore, the preSET and/or postSET domains appear not to be essential for enzymatic activity. Moreover, a new HMT with specificity to H3-K4, which contain neither preSET nor postSET domains was isolated from HeLa cells (Y. Zhang unpubl.). Moreover, an HMT isolated from HeLa cells with specificity for nucleosomal H4-K20 is also devoid of the preSET and the postSET domains (D. Reinberg, unpubl.). Therefore, it is likely that the SET domain is the only signature motif for HMTs. The function of the adjacent cysteine-rich preSET and postSET domains might be replaced by other motifs or might be dispensable if the SET-domain containing HMT exists in a protein complex. For example, the SET domain of Clr4 is sufficient for HMT activity in vitro; however, the chromodomain and the SET domains are both required for activity in vivo (Nakayama et al. 2001). The precise domain requirement for HMT activity and the role of preSET and postSET in histone methylation await further studies.

Coupling histone methylation with DNA methylation The fact that HMTs like CLLD8 and SETDB1/ ESET contain a potential methyl-CpG-binding domain (MBD) raises the important possibility that histone methylation, similar to histone deacetylation (Bird and Wolffe 1999), might function in concert with DNA methylation. As outlined in Figure 6, different modes might be used to couple DNA and histone methylation processes. Methyl-CpG-binding proteins may recruit histone deacetylase complexes to deacetylate histone tails so that the tails become suitable for serving as substrates for methylation (Fig. 6A). In contrast with this sequential process, MBD-containing HMTs may bind directly to methylated nucleosomal DNA to methylate histone tails (Fig. 6B). Alternatively, it is also possible that chromodomain-containing proteins bind to methylated histone tails and recruit DNA methyltransferase (DNMT) to methylate adjacent CpG sequences (Fig. 6C). Irrespective of the sequence of events, it is likely that a concerted action of HMT and HDAC complexes may play an important role in methylated DNA silencing (Fig. 6).

Chromodomain, H3-K9 methylation, and heterochromatin formation Post-translational modifications have long been known to affect protein-protein interactions. For example, phosphorylation on the tumor suppressor protein $\mathrm{Rb}$ affects its interaction with the transcription factor E2F (Harbour and Dean 2000). Recent studies have revealed that acetylation on a protein also affects its association with other proteins. For example, the nuclear hormone receptor coactivator ACTR can be acetylated by $\mathrm{p} 300 / \mathrm{CBP}$ and this modification disrupts the association of the ACTR complex with promoter-bound estrogen receptors (Chen et al. 1999b). In a similar manner, histone modifications have been proposed to serve as markers, termed the histone code, that are recognized by 

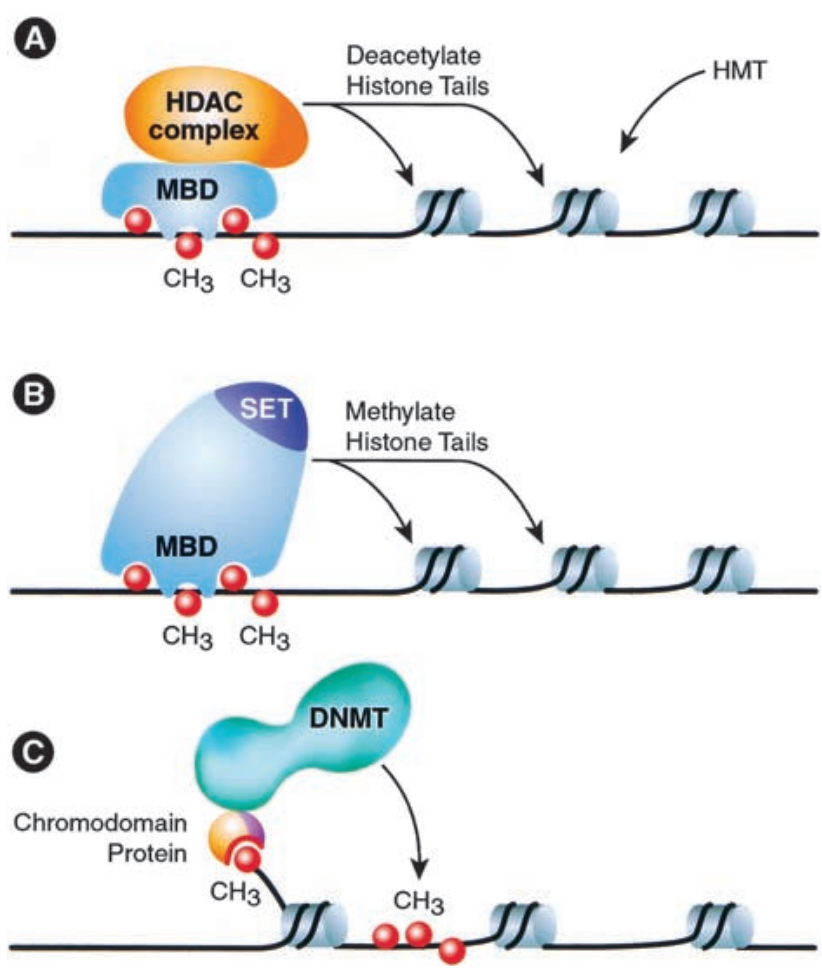

Figure 6. Models depicting the relationship between DNA methylation, histone deacetylation, and histone methylation. (A) Methyl-CpG-binding proteins recruit HDAC complex to deacetylate histone so that the histone tails will be suitable for subsequent methylation by HMTs. (B) In chromatin domains where histones are hypoacetylated, the MBD domain-containing HMTs may bind directly and methylate the histones. $(C)$ Methylated histone tails may recruit DNMTs to methylate DNA for long-term gene silencing.

other proteins (Strahl and Allis 2000; Turner 2000). One piece of evidence that supports this hypothesis is the demonstration that the bromodomain and double bromodomain of the histone acetyltransferases PCAF and TAF250, respectively, bind preferentially to specific acetylated lysines on histone tails (Dhalluin et al. 1999; Jacobson et al. 2000). The ability to recognize modified histone tails does not seem to be limited to the bromodomain. Recent demonstration that the chromodomain present in the heterochromatin protein HP1 binds specifically to methylated H3-K9 further supports the histone code hypothesis (Bannister et al. 2001; Lachner et al. 2001; Nakayama et al. 2001).

Previous studies have indicated that the heterochromatin-associated protein HP1 and its S. pombe homolog Swi6p are required for heterochromatin formation. Importantly, HP1 colocalizes with SUV39H1 (Aagaard et al. 1999). In addition, heterochromatin localization of Swi6p requires functional Clr4 (Ekwall et al. 1996). The chromodomains in the HP1/Swi6p proteins have been implicated in their heterochromatic localization (Platero et al. 1995). These observations collectively suggest that the chromodomain of $\mathrm{HP} 1 / \mathrm{Sw}$ i6p recognizes the $\mathrm{H} 3-\mathrm{K} 9$ methylated by SUV39H1/Clr4. Three groups have re- cently provided supporting evidence for this possibility (Bannister et al. 2001; Lachner et al. 2001; Nakayama et al. 2001). The binding is highly specific because a K9methylated $\mathrm{H} 3$ peptide, but not a K4-methylated peptide displaced HP1 and, importantly, other chromodomaincontaining proteins, including SUV39H1 and Mi-2, failed to bind to methylated H3-K9 peptide. Interestingly, one HP1 chromodomain mutant (V23M) that disrupts the methylated $\mathrm{K} 9$-specific binding activity also disrupts the gene silencing function of Drosophila HP1 (Platero et al. 1995). These findings collectively suggest a correlation between HP1 binding to methylated H3-K9 and its ability to repress transcription. Consistent with the finding that the localization of Swi6p to heterochromatin requires the HMT activity of Clr4 in S. pombe (Bannister et al. 2001; Nakayama et al. 2001), HP1 localization was found to be dispersed in Suv39h1-null PMEFs. Importantly, the dispersed localization of HP1 in the mutant cells could be rescued to heterochromatin foci by retroviral expression of the SUV39H1 (Lachner et al. 2001). Thus, methylation of H3-K9 can serve as a recognition site for the chromodomain of $\mathrm{HP} 1 / \mathrm{Swi}$ p. It is important, however, to point out that although the chromodomain of HP1 has affinity for methylated histone H3-K9, it appears that alternative pathways for HP1 recruitment also exist (Fig. 7 , and see below).

The molecular events leading to the formation of heterochromatin at the centromere and mating-type loci have been recently addressed (Nakayama et al. 2001). Chromatin immunoprecipitation experiments demonstrated that Clr4 preferentially methylates H3-K9 at heterochromatin-associated regions in $S$. pombe. In addition to the SET domain, the chromodomain of Clr4 was found to be required for $\mathrm{H} 3-\mathrm{K} 9$ methylation in vivo. Importantly, mutations in clr3, a gene encoding a H3-specific histone deacetylase, impaired H3-K9 methylation and heterochromatin localization of Swi6 (Nakayama et al. 2001). These important observations suggest that histone deacetylation functions upstream of H3-K9 methylation and Swi6 localization. Because mutations in Clr3 affected specifically histone H3-K14 acetylation levels, deacetylation of K14 seems to be a pre-requirement for H3-K9 methylation. Because acetylation on H3-K9 prevents this site from being methylated (Rea et al. 2000), deacetylation of $\mathrm{H} 3-\mathrm{K} 9$ is also a prerequisite for methylation. As discussed above, Clr4 is required for the localization of Swi6 to heterochromatin (Ekwall et al. 1996). However, Swi6 mutations do not cause any detectable changes in methylation of H3-K9 (Nakayama et al. 2001). Therefore, Swi6 likely functions downstream of Clr4. On the basis of the above observations, a temporal event for heterochromatin formation can be inferred (Fig. 7A). After removal of the acetyl groups from K9 and K14 of histone $\mathrm{H} 3$ by histone deacetylases, Suv39h1/Clr4 methylates H3-K9. Methylated H3-K9 serves as a binding site for the recruitment of HP1/Swi6. HP1/Swi6, through the shadow chromodomain located at its carboxyl terminus, can oligomerize to form heterochromatin (Brasher et al. 2000). One piece of evidence that supports this model is the finding that Swi6 remains asso- 


\section{A Heterochromatin Silencing}

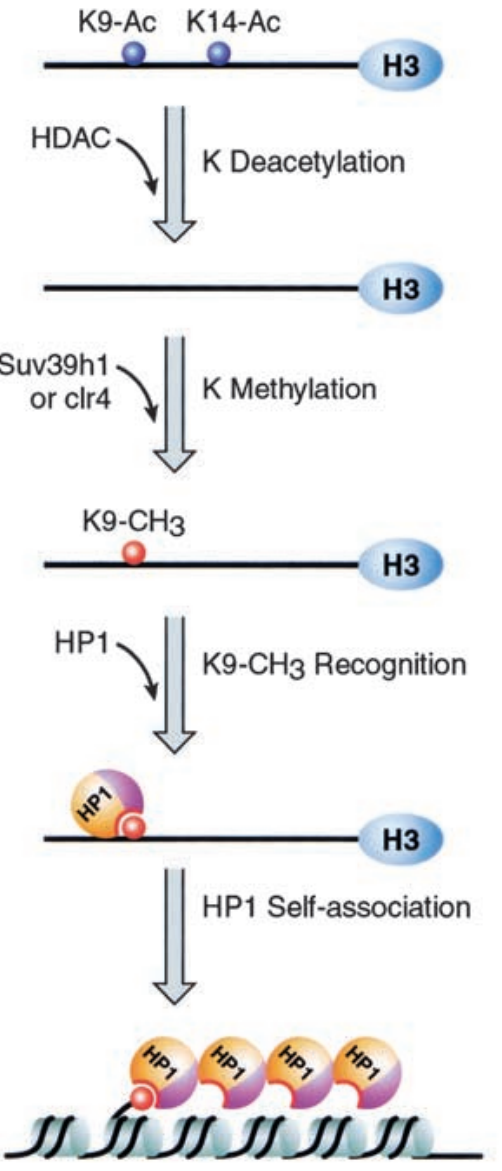

\section{B Euchromatin} Silencing

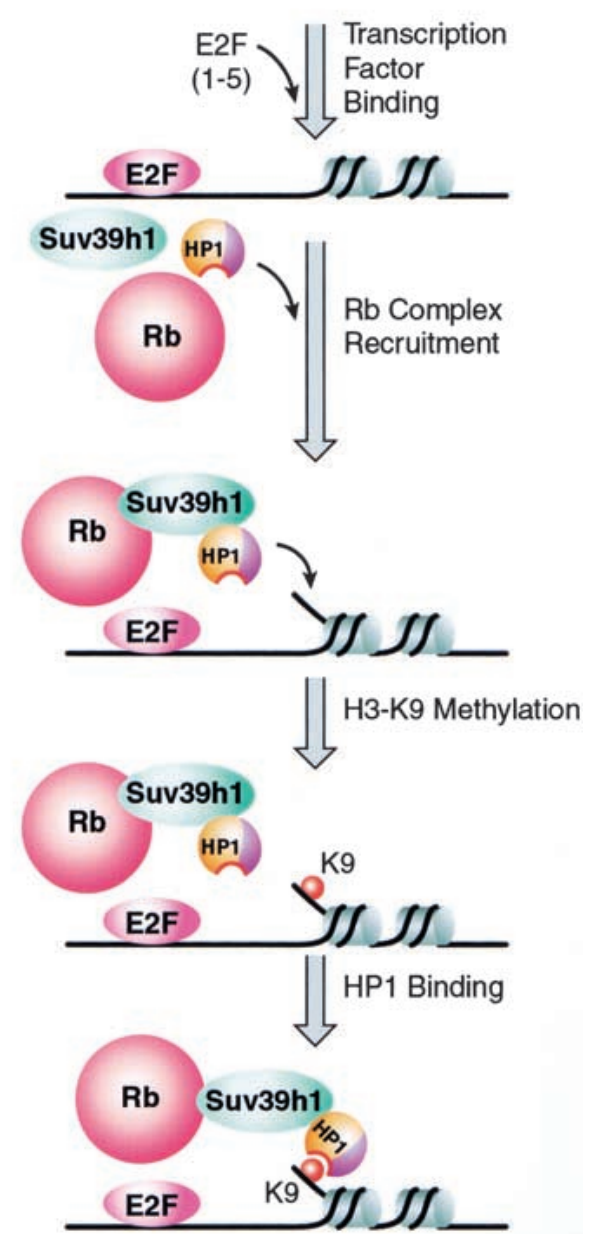

Figure 7. Stepwise models for HP1 in heterochromatin and euchromatin gene silencing. (A) Heterochromatin formation. Acetyl groups are removed from lysine residues by HDACs before Suv39h1/Clr4 can add a methyl group to Lys 9. The chromodomain of HP1 recognizes and binds to methyl-K9 to initiate heterochromatin formation. The heterochromatin is propagated by self-association of HP1 through its shadow chromodomain. In addition to recognizing methyl-K9, HP1 can also bind to the globular domain of H3. (B) Silencing of euchromatic genes by HP1. Transcription factors, such as E2F, recruit Suv39h1/ HP1 complex through the tumor suppressor $\mathrm{Rb}$ to a specific gene promoter. After methylation of H3-K9 by Suv39h1, HP1 binds and represses transcription. ciated with the mating-type locus mat $2 / 3$ throughout the cell cycle (Nakayama et al. 2000). This finding suggests that Swi6 might play an important role in the inheritance of the silenced state of the heterochromatin. Importantly, it is currently unknown whether oligomerization of $\mathrm{Hp} 1 /$ Swi6 requires $\mathrm{H} 3-\mathrm{K} 9$ methylation at each nucleosome.

The studies described above demonstrated an important role for H3-K9 methylation in the recruitment of Swi6/HP1 and heterochromatin silencing. However, evidence supporting alternative pathways for HP1 recruitment does exist. For example, it was recently demonstrated that HP1 interacts with the globular histone fold domain of $\mathrm{H} 3$ as well as with the linker histone $\mathrm{H} 1$ through the chromodomain and hinge region, respectively (A.L. Nielsen et al. 2001). Because HP1 can oligomerize through its shadow chromodomain (Brasher et al. 2000), it is possible that the chromodomains of the HP1 oligomers can also interact with the histone fold.

In addition to heterochromatin silencing, recent studies indicate that HP1 is involved in silencing of specific genes in euchromatin (Fig. 7B). It was found that Rb, through its pocket domain, interacts with HP1 and
SUV39H1 in vivo to regulate the expression of the cyclin $E$ gene (S.J. Nielsen et al. 2001). Targeting of $\mathrm{Rb}$ to the cyclin $E$ gene occurs through the transcription factor E2F. The expression level of cyclin $E$ is elevated in SUV39H1-disrupted fibroblasts. Chromatin immunoprecipitation experiments indicate that $\mathrm{Rb}$ is required for methylation of $\mathrm{H} 3-\mathrm{K} 9$ as well as for $\mathrm{HP} 1$ recruitment to the cyclin E promoter (S.J. Nielsen et al. 2001). In contrast to the spreading of H3-K9 methylation and HP1 binding in heterochromatin, Rb-mediated SUV39H1/ HP1 recruitment and H3-K9 methylation occurred within one nucleosome surrounding the cyclin $E$ promoter (S.J. Nielsen et al. 2001). Recruitment of histone deacetylases by $\mathrm{Rb}$ was shown previously to be part of the mechanism by which $\mathrm{Rb}$ mediates transcription repression (Brehm et al. 1998; Luo et al. 1998; MagnaghiJaulin et al. 1998). The involvement of HMT activity in $\mathrm{Rb}$-mediated transcription repression suggests again that HDACs and HMTs may work in concert in the silencing of euchromatic genes. Moreover, recent studies with E2F-6, a member of the E2F-family of transcription factors that functions in $\mathrm{G}_{0}$ in an $\mathrm{Rb}$-independent manner, demonstrate that E2F-6 also exists in a protein complex 
with HP1 and an HMT (D. Livingston and Y. Nakatani, pers. comm.). Whether this complex also contains an HDAC remains to be determined. However, the above studies collectively illustrate alternative pathways by which HP1 can be recruited to different genes to silence their expression. More importantly, the studies with $\mathrm{Rb}$ regulating the expression of cyclin $E$ though nucleosome methylation strongly suggest that this modification must be removed at every cell cycle event. Whether this removal is mediated by histone demethylation, the processing of the histone polypeptide, and/or histone degradation is an important open question (see below).

Can lysine methylation be a mark for transcription activation? Although the studies described above indicate that H3-K9 methylation functions as a repressive mark, not all lysine methylation appears to be a signal for repression of transcription. It appears that a particular modification, such as methylation at $\mathrm{H} 3-\mathrm{K} 9$, might have a different readout depending on its chromosomal location and combination of modifications and the enzyme (or protein complex) involved in the particular modification. A recent report has suggested that the histone acetyltransferase p300/CBP interacts with an H3 histone methyltransferase with specificity for H3-K9 (Vandel and Trouche 2001), suggesting that H3-K9 methylation could potentially be involved in transcriptional activation as well. Previous studies have indicated that a SET domain-containing protein ASH1 (absent, small, and homeotic discs) is able to interact with CBP (Bantignies et al. 2000) suggesting that the p300/CBP-associated histone methyltransferase is likely to be the mammalian homolog of Drosophila ASH1. ASH1 was recently found to be capable of methylating multiple lysine residues, including histone H3-K4 and H3-K9 and histone H4-K20. Importantly, a single amino acid substitution in the SET domain crippled the HMT enzymatic activity as well as ASH1-dependent transcriptional activation (Frank Sauer, pers. comm.). Therefore, at least four different enzymes Suv39h1, Suv39h2, G9a (O'Carroll et al. 2000; Rea et al. 2000; Tachibana et al. 2001) and ASH1 have been shown to methylate H3-K9. Of these four enzymes, only Suv39h1 and Suv39H2 are specific for H3-K9, the other two enzymes, G9a and ASH1 apparently can methylate H3-K9 in combination with other residues. Therefore, it is possible that methylation at $\mathrm{H} 3-\mathrm{K} 9$ alone results in transcription repression, whereas methylation at $\mathrm{H} 3-\mathrm{K} 9$ together with methylation at H3-K27 (by G9a, see above) or methylation at these two residues in combination with methylation at $\mathrm{H} 4-\mathrm{K} 20$ might result in transcription activation. In support of the hypothesis that methylation at $\mathrm{H} 3-\mathrm{K} 4$ can result in transcription activation, $\mathrm{H} 3-\mathrm{K} 4$ was found to be a preferred site of methylation in the transcriptionally active macronuclei of Tetrahymena, and importantly, methylation at H3-K4 correlates with acetylation (Strahl et al. 1999). Moreover, recent studies have found that the yeast Set- 1 protein, which apparently targets H3-K4, exists in a complex with transcriptional activators (T. Kouzarides, pers. comm.). Therefore, it is likely that methylation at H3-K4 independently, or in combination with methylation at H3K9, results in transcriptional activation. The mechanism(s) by which methylation at $\mathrm{H} 3-\mathrm{K} 4$ results in transcriptional activation is unknown, but it is possible that methylation at these residues can mark a gene for the recruitment of complexes involved in activation and/or can displace complexes involved in transcriptional repression, such as histone deacetylases.

A recent study by Grewal and colleagues demonstrates that euchromatic and heterochromatic chromosomal domains have distinctive site-specific H3 methylation patterns (Noma et al. 2001). Chromatin immunoprecipitation (ChIP) experiments with antibodies specific to H3K9 and H3-K4 and expanding the fission yeast matingtype locus have revealed that $\mathrm{H} 3-\mathrm{K} 9$ methylation is strictly localized to a $20-\mathrm{kb}$ silent heterochromatic region, whereas $\mathrm{H} 3-\mathrm{K} 4$ methylation is specific to the surrounding euchromatic regions (Fig. 8). Importantly, two inverted repeats that flank the silent interval serve as boundary elements for heterochromatin and euchromatin as deletions of these boundary elements lead to spreading of H3-K9 methylation into the neighboring se-

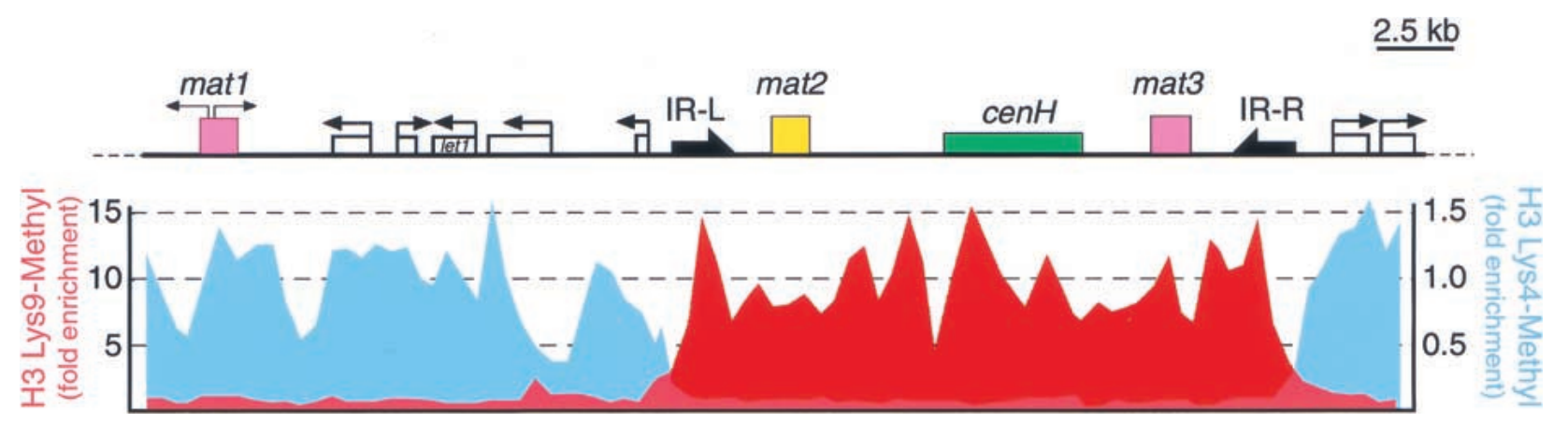

Figure 8. Differential sites of histone $\mathrm{H} 3$ methylation mark euchromatic and heterochromatic regions at the fission yeast mat locus. (Top) Physical map of the mating-type region. IR-L and IR-R, shown as black arrows, denote inverted repeats residing on both sides of the mat2/3 interval and within is $c e n H$, with homology to centromeric repeats. (Boxes) Open reading frames, including the essential let $1^{+}$gene; (arrows) direction of transcription. The graph represents a summary of ChIP experiments throughout the mat locus with H3-K4 (blue) and H3-K9 (red) methyl-specific antibodies (for details, see Noma et al. 2001). Courtesy of Dr. Shiv Grewal. 
quences (Noma et al. 2001). These studies collectively support the idea that site-specific methylation can serve as chromatin domain markers.

\section{Interplay between different histone tail modifications}

As discussed above, histone tails are rich in residues that can be covalently modified (Fig. 1). Increasing evidence indicates that the different modifications can affect each other (Fig. 9). For example, several transcription-related HATs including GCN5, PCAF, and p300, prefer S10 phosphorylated H3 as a substrate (Cheung et al. 2000; Lo et al. 2000).

Studies in Tetrahymena demonstrated that phosphorylation of H3-S10 is required for proper chromosome condensation and segregation (Wei et al. 1999). The enzymes responsible for adding and removing the phosphate group from H3-S10 were identified as the Ipl1p/ aurora-2 kinase and Glc7p/PP1 phosphatase, respectively (Hsu et al. 2000). Recent studies have demonstrated, however, that phosphorylation of H3-S10 also occurs during interphase, and importantly it correlates with transcriptional activation. The RSK-2 kinase was found to be activated in response to different effectors resulting in the phosphorylation of different transcription factors as well as phosphorylation of histone H3-S10 (Sassone-Corsi et al. 1999). Moreover, recent studies in yeast have revealed that the Snf1 kinase phosphorylates histone $\mathrm{H} 3-\mathrm{S} 10$ and that this phosphorylation is required for transcriptional activation of certain yeast genes (Lo et al. 2001). These findings collectively demonstrate that more than one kinase is involved in H3-S10 phosphorylation and that the phosphorylation status of H3-S10 plays an important role in transcriptional regulation.

H3-S10 phosphorylation facilitates GCN5-mediated acetylation of H3-K14 (Cheung et al. 2000; Lo et al. 2000). By use of antibodies that specifically recognize phosphorylated H3-S10 or acetylated H3-K14, as well as a specific antibody recognizing both modifications, it was uncovered that following EGF treatment, the accumulation of singly phosphorylated H3 preceded H3-K14 acetylation (Cheung et al. 2000). A simple explanation for this observation is that phosphorylation is followed by acetylation to generate the dimodified H3 (Fig. 9). These sequential events were also observed in yeast. It was found that phosphorylation of H3-S10 promotes subsequent acetylation of H3-K14 by GCN5. Importantly, acetylation of H3-K14 had no effect on phosphorylation of histone H3-S10 (Lo et al. 2000). Moreover, a mutation in the GCN5 HAT domain (R164A) selectively crippled H3-S10 phosphorylation-enhanced acetylation whereas the mutation had no significant affect on the acetylation of nonphosphorylated substrate. Importantly, this mutation also affected the activity of a subset of GCN5-regulated promoters in vivo (Lo et al. 2000). These results suggest that the enhanced acetylation of H3-K14 mediated by phosphorylation of H3-S10 plays an important role in transcription of a subset of GCN5-regulated genes.

In addition to the interplay observed between H3-S10 phosphorylation and H3-K14 acetylation, phosphorylation on histone H3-S10 also plays an important role in regulating lysine methylation. It was recently reported that methylation of histone H3-K9 by SUV39H1 was greatly inhibited when an $\mathrm{H} 3$-tail peptide phosphorylated at S10 was used as a substrate (Rea et al. 2000). Similarly, methylation on H3-K9 inhibits subsequent phosphorylation of H3-S10. As expected, acetylation and methylation of histone H3-K9 are mutually exclusive (Fig. 9). This observation is not surprising as methylation of H3-K9 correlates with gene silencing, whereas acetylation of histone H3-K9 correlates with transcriptional activation. The interplay observed between phosphorylation and acetylation is consistent with the findings that activation of different signal transduction pathways, resulting in the transcription of specific genes

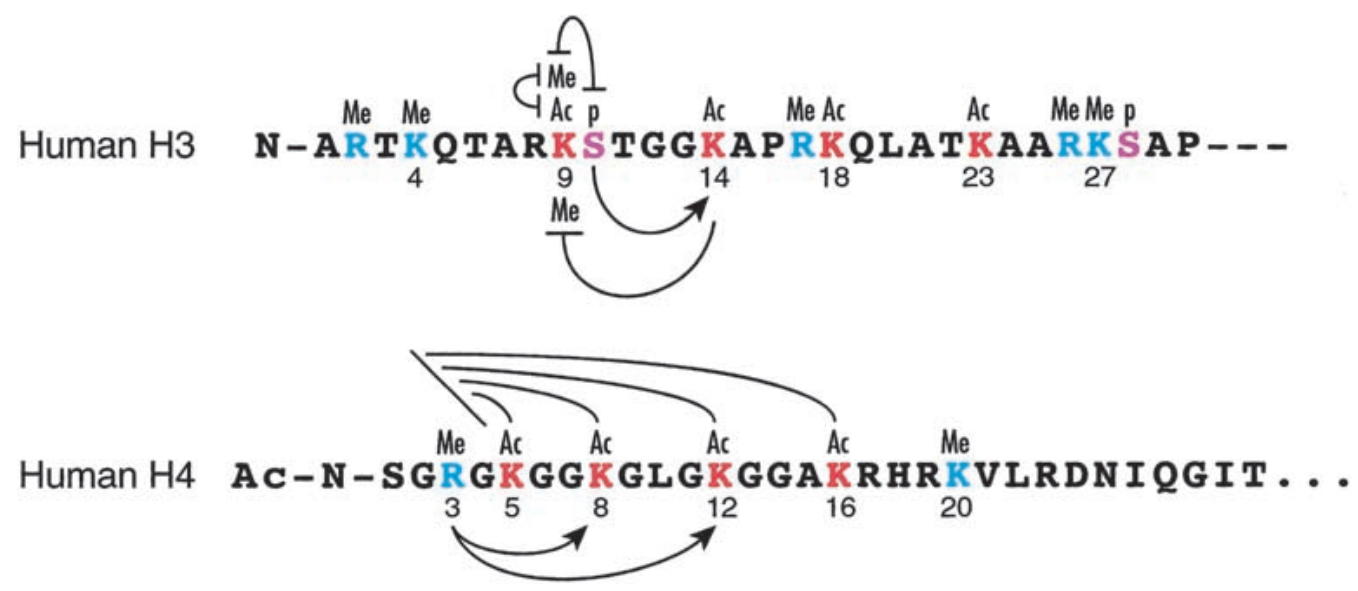

Figure 9. Interplay between different post-translational modifications occurring on histone $\mathrm{H} 3$ and $\mathrm{H} 4$ amino-terminal tails. Residues that are known to be acetylated $(\mathrm{Ac})$, methylated $(\mathrm{Me})$, and phosphorylated $(\mathrm{P})$ are indicated. Positive and negative affects are indicated. 
correlates with phosphorylation of histone H3-S10. Phosphorylation on H3-S10, in turn, inhibits methylation of histone H3-K9, and promotes acetylation of histone $\mathrm{H} 3-\mathrm{K} 14$, a modification present on transcriptionally active genes.

Similar to histone $\mathrm{H} 3$, multiple modifications also exist on the histone $\mathrm{H} 4$ tail. In addition to the well-characterized lysine acetylation (Lys 5, Lys 8, Lys 12, and Lys 16), methylation at Lys 20 and phosphorylation at Ser 1 have also been documented (Strahl and Allis 2000; Fig. 1). Adding to this complexity is the recent discovery that H4-R3 can also be methylated by PRMT1 (see above and Wang et al. 2001; Strahl et al. 2001). Significantly, methylation on H4-R3 facilitates acetylation by p300 on histone H4-K8 and H4-K12 (Fig. 9). Consistent with a role in facilitating p300-mediated lysine acetylation, the methyltransferase activity of PRMT1 was found to stimulate transcription (Wang et al. 2001). The interplay between H4-R3 methylation and lysine acetylation is not unidirectional. Lysine acetylation can also affect subsequent H4-R3 methylation (Fig. 9). It was found that acetylation on any of the four lysines on the histone $\mathrm{H} 4$ aminoterminal tail inhibits subsequent methylation on H4-R3 by PRMT1 (Wang et al. 2001). On the basis of the relationship between H4-R3 methylation and lysine acetylation, it is likely that H4-R3 methylation precedes lysine acetylation.

The histone code hypothesis predicts that different functional domains present in different polypeptides recognize different histone modifications. Thus far, acetyllysines and methyl-lysine 9 have been found to be recognized by bromodomain- (Dhalluin et al. 1999; Jacobson et al. 2000) and chromodomain-containing polypeptides (Bannister et al. 2001; Lachner et al. 2001; Nakayama et al. 2001), respectively. This recognition appears to be highly specific, as not all chromodomaincontaining proteins bind to methylated $\mathrm{H} 3-\mathrm{K} 9$. It is likely that the residues within and surrounding the chromodomain determine specificity. Understanding the function of the different modifications occurring on the histone tails will be greatly facilitated by elucidating the domains responsible for recognition of modifications occurring at other residues (H3-K4methyl, H3-S10-phospho, H4-R3-methyl, and H4-K20methyl). Because methylation on histone H4-R3 makes $\mathrm{H} 4$ a better substrate for p300-mediated acetylation (Wang et al. 2001), methylated H4-R3 might be a recognition signal for the bromodomain of $\mathrm{p} 300$. Three arginines of the histone $\mathrm{H} 3$ amino-terminal tails (Arg 2, Arg 17, and Arg 26) were recently shown to be methylated by CARM1 in vitro (Schurter et al. 2001). Therefore, 11 of 28 amino acids of the histone H3-tail are subject to one or two types of post-translational modification (Fig. 1). Given the interplay between different modifications, which theoretically can occur within or between different histone tails, the combinations of modifications with a mononucleosome is enormous. Moreover, some of these modifications are likely to be regulated by regions within chromosomes. Therefore, the interplay between the different modifica- tions directly contributes to the diversity of the histone code.

\section{Future directions}

Histone methylation was discovered three decades ago. Its functional significance has just begun to be addressed. The discovery of the first HAT was instrumental in dissecting the function of histone acetylation. Similarly, the discovery of CARM1, SUV39H1, and PRMT1 as HMTs will greatly help our understanding of the function of histone methylation. We anticipate a flurry of research isolating and characterizing HMTs as well as defining genes or gene complexes that are regulated by specific HMTs. The interplay between HMTs, histone acetylation and histone phosphorylation in regulating specific set of genes is forthcoming.

As discussed in this review, two families of HMTs with distinct substrate (arginine and lysine) and site specificity have been discovered. The known functions of these two family of proteins predict that histone methylation is likely to play important roles in multiple biological processes including transcription, signal transduction, development, and cellular proliferation and differentiation. To understand how histone methylation participates in the above biological processes, progress in several areas of histone methylation study are needed. First, the sites of histone methylation need to be further characterized in vivo. Although some of the major sites for $\mathrm{H} 3$ and $\mathrm{H} 4$ are known, the methylation sites for $\mathrm{H} 2 \mathrm{~A}$ and $\mathrm{H} 2 \mathrm{~B}$ are poorly characterized. With the use of more sensitive detection methods, we anticipate that novel methylation sites will be discovered. Second, the functional significance for each methylated residue needs to be determined. The generation of antibodies specifically recognizing modified residues will provide an important tool in this regard. Third, the use of biochemical purification and sequence similarity approaches will likely lead to the discovery of novel HMTs, some of which are likely to contain unique catalytic domains other than the SET domain or the conserved arginine methyltransferase motifs. An important issue is the identification of HMTs that can use nucleosomal histones as substrates. It is also important to know whether nucleosome remodeling is required for HMTs to methylate nucleosomal histones.

Unlike the dynamic process of histone acetylation, histone methylation is believed to be relatively static (Shepherd et al. 1971; Byvoet et al. 1972). However, changes in histone methylation levels during the cell cycle or in response to heat shock have been reported (Camato and Tanguay 1982; Annunziato et al. 1995). Whether enzymes responsible for the removal of the methyl group from histones exist still remains to be determined. If histone demethylases (HDMs) do not exist, cells would have had to develop systems to deal with the consequence of this modification. For example the methylated histones could be more susceptible to degradation so that histone methylation can be regulated. Enzymes with demethylase activity have been previously reported 
(J. Lee et al. 1996). However, enzymes capable of actively demethylating histone polypeptides remain to be discovered. Characterization of the HMTs and HDMs will have great impact on the study of many biological processes in which histones play a role. It is likely that histone methylation, like DNA methylation, may mark genes, or chromosomal regions. Whether these marked regions can become transcriptionally active through active demethylation or processes requiring repair or coupled to protein degradation remains an extremely important question. Of interest are earlier observations suggesting that the tails of histone H3 (Allis et al. 1980) and H4 (Lin et al. 1991) are subject to proteolytic processing and that a ubiquitin-specific protease suppresses variegation in Drosophila (Henchoz et al. 1996).

\section{Acknowledgments}

We thank David Allis, Shelley Berger, Tony Kouzarides, Yoshihiro Nakatani, Paolo Sassone-Corsi, Frank Sauer, and Liu Yang for communication of unpublished results. We also thank Drs. Xiaodong Cheng and Shiv Grewal for providing Figures 4 and 8 , respectively. We also thank Drs. Marc Gartenberg, Michael Hampsey, and Yoshihiro Nakatani for helpful comments on the manuscript. Work in D.R.'s laboratory is supported by grants from the National Institutes of Health (GM37120 and GM48518) and the Howard Hughes Medical Institute. Y.Z. is a Kimmel Scholar and work in his laboratory is supported by grants from NIH (GM63067) and ACS (RSG-01-233-01-GMC).

\section{References}

Aagaard, L., Laible, G. Selenko, P., Schmid, M., Dorn, R., Schotta, G., Kuhfittig, S., Wolf, A., Lebersorger, A., Singh, P.B., et al. 1999. Functional mammalian homologues of the Drosophila PEV-modifier Su(var)3-9 encode centromere-associated proteins which complex with the heterochromatin component M31. EMBO J. 18: 1923-1938.

Abramovich, C., Yakobson, B., Chebath, J., and Revel, M. 1997. A protein-arginine methyltransferase binds to the intracytoplasmic domain of the IFNAR1 chain in the type I interferon receptor. $E M B O ~ J$. 16: 260-266.

Aletta, J.M., Cimato, T.R., and Ettinger, M.J. 1998. Protein methylation: A signal event in post-translational modification. Trends Biochem. Sci. 23: 89-91.

Allis, C.D., Bowen, J.K., Abraham, G.N., Glover, C.V., and Gorovsky, M.D. 1980. Proteolytic processing of histone H3 in chromatin: A physiologically regulated event in Tetrahymena micronuclei. Cell 20: 55-64.

Annunziato, A.T., Eason, M.B., and Perry, C.A. 1995. Relationship between methylation and acetylation of arginine-rich histones in cycling and arrested HeLa cells. Biochemistry 34: 2916-2924.

Bannister, A.J., Zegerman, P., Partridge, J.F., Miska, E.A., Thomas, J.O., Allshire, R.C., and Kouzarides, T. 2001. Selective recognition of methylated lysine 9 on histone $\mathrm{H} 3$ by the HP1 chromodomain. Nature 410: 120-124.

Bantignies, F., Goodman, R.H., and Smolik, S.M. 2000. Functional interaction between the coactivator Drosophila CREB-binding protein and ASH1, a member of the trithorax group of chromatin modifiers. Mol. Cell. Biol. 20: 93179330 .
Bird, A.P. and Wolffe, A.P. 1999. Methylation-induced repressio-belts, braces, and chromatin. Cell 99: 451-454.

Branscombe, T.L., Frankel, A., Lee, J.H., Cook, J.R., Yang, Zh-Z., Pestka, S., and Clarke, S. 2001. PRMT5 (the Janus KinaseBinding Protein 1) catalyzes the formation of symmetric dimethylarginine residues in proteins. J. Biol. Chem. 2001 Jun 18 [epub ahead of print].

Brasher, S.V., Smith, B.O., Fogh, R.H., Nietlispach, D., Thiru, A., Nielsen, P.R., Broadhurst, R.W., Ball, L.J., Murzina, N.V., and Laue, E.D. 2000. The structure of mouse HP1 suggests a unique mode of single peptide recognition by the shadow chromodomain dimer. EMBO J. 19: 1587-1597.

Brehm, A., Miska, E.A., McCance, D.J., Reid, J.L., Bannister, A.J., and Kouzarides, T. 1998. Retinoblastoma protein recruits histone deacetylase to repress transcription. Nature 391: 597-601.

Brownell, J.E., Zhou, J., Ranalli, T., Kobayashi, R., Edmondson, D.G., Roth, S.Y., and Allis, C.D. 1996. Tetrahymena histone acetyltransferase A: A homolog to yeast Gcn5p linking histone acetylation to gene activation. Cell 84: 843-851.

Byvoet, P., Shepherd, G.R., Hardin, J.M., and Noland, B.J. 1972. The distribution and turnover of labeled methyl groups in histone fractions of cultured mammalian cells. Arch. Biochem. Biophys. 148: 558-567.

Camato, R. and Tanguay, R.M. 1982. Changes in the methylation pattern of core histones during heat-shock in Drosophila cells. EMBO J. 1: 1529-1532.

Chen, D., Huang, S.M., and Stallcup, M.R. 2000. Synergistic, p160 coactivator-dependent enhancement of estrogen receptor function by CARM1 and p300. J. Biol. Chem. 275: 40810-40816.

Chen, D., Ma, H., Hong, H., Koh, S.S., Huang, S.M., Schurter, B.T., Aswad, D.W., and Stallcup, M.R. 1999a. Regulation of transcription by a protein methyltransferase. Science 284: 2174-2177.

Chen, H., Lin, R.J., Xie, W., Wilpitz, D., and Evans, R.M. 1999b. Regulation of hormone-induced histone hyperacetylation and gene activation via acetylation of an acetylase. Cell 98: 675-686.

Cheung, P., Tanner, K.G., Cheung, W.L., Sassone-Corsi, P., Denu, J.M. and Allis, C.D. 2000. Synergistic coupling of histone $\mathrm{H} 3$ phosphorylation and acetylation in response to epidermal growth factor stimulation. Mol. Cell 5: 905-915.

Clarke, S. 1993. Protein methylation. Curr. Opin. Cell Biol. 5: 977-983.

Dhalluin, C., Carlson, J.E., Zeng, L., He, C., Aggarwal, A.K., and Zhou, M.M. 1999. Structure and ligand of a histone acetyltransferase bromodomain. Nature 399: 491-496.

Ekwall, K., Nimmo, E.R., Javerzat, J.P., Borgstrom, B., Egel, R., Cranston, G., and Allshire, R. 1996. Mutations in the fission yeast silencing factors clr4+ and rik1+ disrupt the localisation of the chromodomain protein Swi6p and impair centromere function. J. Cell Sci. 109: 2637-2648.

Frankel, A. and Clarke, S. 2000. PRMT3 is a distinct member of the protein arginine $\mathrm{N}$-methyltransferase family. Conferral of substrate specificity by a zinc-finger domain. J. Biol. Chem. 275: 32974-32982.

Gary, J.D. and Clarke, S. 1998. RNA and protein interactions modulated by protein arginine methylation. Prog. Nucleic Acid Res. Mol. Biol. 61: 65-131.

Gary, J.D., Lin, W.J., Yang, M.C., Herschman, H.R., and Clarke, S. 1996. The predominant protein-arginine methyltransferase from Saccharomyces cerevisiae. J. Biol. Chem. 271: 12585-12594.

Gilbreth, M., Yang, P., Bartholomeusz, G., Pimental, R.A., Kansra, S., Gadiraju, R., and Marcus, S. 1998. Negative regula- 
tion of mitosis in fission yeast by the shk1 interacting protein skb1 and its human homolog, Skb1Hs. Proc. Natl. Acad. Sci. 95: 14781-14786.

Grunstein, M. 1998. Yeast heterochromatin: Regulation of its assembly and inheritance by histones. Cell 93: 325-328.

Grunstein, M., Hecht, A., Fisher-Adams, G., Wan, J., Mann, R.K., Strahl-Bolsinger, S., Laroche, T., and Gasser, S. 1995. The regulation of euchromatin and heterochromatin by histones in yeast. J. Cell Sci. (Suppl.) 19: 29-36.

Harbour, J.W. and Dean, D.C. 2000. The Rb/E2F pathway: Expanding roles and emerging paradigms. Genes \& Dev. 14: 2393-2409.

Harte, P.J., Wu, W., Carrasquillo, M.M., and Matera, A.G. 1999. Assignment of a novel bifurcated SET domain gene, SETDB1, to human chromosome band 1q21 by in situ hybridization and radiation hybrids. Cytogenet. Cell Genet. 84: 83-86.

Hassig, C.A., Tong, J.K., Fleischer, T.C., Owa, T., Grable, P.G., Ayer, D.E., and Schreiber, S.L. 1998. A role for histone deacetylase activity in HDAC1-mediated transcriptional repression. Proc. Natl. Acad. Sci. 95: 3519-3524.

Hayes, J.J. and Hansen, J.C. 2001. Nucleosomes and the chromatin fiber. Curr. Opin. Genet. Dev. 11: 124-129.

Hebbes, T.R., Thorne, A.W., and Crane-Robinson, C. 1988. A direct link between core histone acetylation and transcriptionally active chromatin. EMBO J. 7: 1395-1402.

Hendrich, B. and Bird, A. 1998. Identification and characterization of a family of mammalian methyl-CpG binding proteins. Mol. Cell. Biol. 18: 6538-6547.

Henchoz, S., De Rubertis, F., Pauli, D., and Spierer, P. 1996. The dose of a putative ubiquitin-specific protease affects position-effect variegation in Drosophila melanogaster. Mol. Cell Biol. 16: 5717-5725.

Henry, M.F. and Silver, P.A. 1996. A novel methyltransferase (Hmtlp) modifies poly(A)+-RNA-binding proteins. Mol. Cell. Biol. 16: 3668-3678.

Hsu, J.Y., Sun, Z.W., Li, X., Reuben, M., Tatchell, K., Bishop, D.K., Grushcow, J.M., Brame, C.J., Caldwell, J.A., Hunt, D.F., et al. 2000. Mitotic phosphorylation of histone $\mathrm{H} 3$ is governed by Ipl1/aurora kinase and Glc7/PP1 phosphatase in budding yeast and nematodes. Cell 102: 279291.

Jacobson, R.H., Ladurner, A.G., King, D.S., and Tjian, R. 2000. Structure and function of a human TAFII250 double bromodomain module. Science 288: 1422-1425.

Jenuwein, T., Laible, G., Dorn, R., and Reuter, G. 1998. SET domain proteins modulate chromatin domains in eu- and heterochromatin. Cell. Mol. Life Sci. 54: 80-93.

Jones, R.S. and Gelbart, W.M. 1993. The Drosophila Polycombgroup gene Enhancer of zeste contains a region with sequence similarity to trithorax. Mol. Cell. Biol. 13: 63576366.

Kadosh, D. and Struhl, K. 1998. Histone deacetylase activity of $\mathrm{Rpd} 3$ is important for transcriptional repression in vivo. Genes \& Dev. 12: 797-805.

Katsanis, N., Yaspo, M.L., and Fisher, E.M. 1997. Identification and mapping of a novel human gene, HRMT1L1, homologous to the rat protein arginine $\mathrm{N}$-methyltransferase 1 (PRMT1) gene. Mamm. Genome 8: 526-529.

Kingston, R.E. and Narlikar, G.J. 1999. ATP-dependent remodeling and acetylation as regulators of chromatin fluidity. Genes \& Dev. 13: 2339-2352.

Klein, R.R. and Houtz, R.L. 1995. Cloning and developmental expression of pea ribulose-1,5-bisphosphate carboxylase/ oxygenase large subunit N-methyltransferase. Plant Mol.
Biol. 27: 249-261.

Koh, S.S., Chen, D., Lee, Y.H., and Stallcup, M.R. 2001. Synergistic enhancement of nuclear receptor function by p160 coactivators and two coactivators with protein methyltransferase activities. J. Biol. Chem. 276: 1089-1098.

Koonin, E.V., Zhou, S., and Lucchesi, J.C. 1995. The chromo superfamily: New members, duplication of the chromodomain and possible role in delivering transcription regulators to chromatin. Nucleic Acids Res. 23: 4229-4233.

Kornberg, R.D. and Lorch, Y. 1999. Twenty-five years of the nucleosome, fundamental particle of the eukaryote chromosome. Cell 98: 285-294.

Kuo, M.H., Zhou, J., Jambeck, P., Churchill, M.E., and Allis, C.D. 1998. Histone acetyltransferase activity of yeast Gcn5p is required for the activation of target genes in vivo. Genes \& Dev. 12: 627-639.

Kuzmichev, A. and Reinberg, D. 2001. Role of histone deacetylase complexes in the regulation of chromatin metabolism. Curr. Top. Microbiol. Immunol. 254: 35-58.

Lachner, M., O'Carroll, D., Rea, S., Mechtler, K., and Jenuwein, T. 2001. Methylation of histone H3 lysine 9 creates a binding site for HP1 proteins. Nature 410: 116-120.

Lee, J., Chen, Y., Tolstykh, T., and Stock, J. 1996. A specific protein carboxyl methylesterase that demethylates phosphoprotein phosphatase 2A in bovine brain. Proc. Natl. Acad. Sci. 93: 6043-6047.

Lee, J.H., Cook, J.R., Pollack, B.P., Kinzy, T.G., Norris, D., and Pestka, S. 2000. Hs17p, the yeast homologue of human JBP1, is a protein methyltransferase. Biochem. Biophys. Res. Commun. 274: 105-111.

Lee, M.S., Henry, M., and Silver, P.A. 1996. A protein that shuttles between the nucleus and the cytoplasm is an important mediator of RNA export. Genes \& Dev. 10: 12331246.

Lin, R., Cook, R.G., and Allis, C.D. 1991. Proteolytic removal of core histone amino termini and dephosphorylation of histone $\mathrm{H} 1$ correlates with the formation of condensed chromatin and transcriptional silencing during Tetrahymena macronuclear development. Genes \& Dev. 5: 1601-1610.

Lin, W.J., Gary, J.D., Yang, M.C., Clarke, S., and Herschman, H.R. 1996. The mammalian immediate-early TIS21 protein and the leukemia-associated BTG1 protein interact with a protein-arginine N-methyltransferase. J. Biol. Chem. 271: 15034-15044.

Lo, W.S., Trievel, R.C., Rojas, J.R., Duggan, L., Hsu, J.Y.,. Allis, C.D, Marmorstein, R., and Berger, S.L. 2000. Phosphorylation of serine 10 in histone $\mathrm{H} 3$ is functionally linked in vitro and in vivo to Gen5-mediated acetylation at lysine 14. Mol. Cell 5: 917-926.

Lo, W.S., Duggan, L., Lane, W., Shiekhattar, R., and Berger, S.L. 2001. Snfl is a histone kinase which works in concert with the histone acetyltransferase Gcn5 to regulate transcription. Science 293: 1142-1146.

Loubbardi, A., Marcireau, C., Karst, F., and Guilloton, M. 1995. Sterol uptake induced by an impairment of pyridoxal phosphate synthesis in Saccharomyces cerevisiae: Cloning and sequencing of the PDX3 gene encoding pyridoxine (pyridoxamine) phosphate oxidase. J. Bacteriol. 177: 18171823.

Luger, K., Mader, A.W., Richmond, R.K., Sargent, D.F., and Richmond, T.J. 1997. Crystal structure of the nucleosome core particle at $2.8 \AA$ A resolution. Nature 389: 251-260.

Luo, R.X., Postigo, A.A., and Dean, D.C. 1998. Rb interacts with histone deacetylase to repress transcription. Cell 92: 463473. 
Ma, X.J., Lu, Q., and Grunstein, M. 1996. A search for proteins that interact genetically with histone $\mathrm{H} 3$ and $\mathrm{H} 4$ amino termini uncovers novel regulators of the Swel kinase in Saccharomyces cerevisiae. Genes \& Dev. 10: 1327-1340.

Mabuchi, H., Fujii, H., Calin, G., Alder, H., Negrini, M., Rassenti, L., Kipps, T.J., Bullrich, F., and Croce, C.M. 2001. Cloning and characterization of CLLD6, CLLD7, and CLLD8, novel candidate genes for leukemogenesis at chromosome 13q14, a region commonly deleted in B-cell chronic lymphocytic leukemia. Cancer Res. 61: 2870-2877.

Magnaghi-Jaulin, L., Groisman, R., Naguibneva, I., Robin, P., Lorain, S., Le Villain, J.P., Troalen, F., Trouche, D., and Harel-Bellan, A. 1998. Retinoblastoma protein represses transcription by recruiting a histone deacetylase. Nature 391: 601-605.

McBride, A.E., Weiss, V.H., Kim, H.K., Hogle, J.M., and Silver, P.A. 2000. Analysis of the yeast arginine methyltransferase Hmtlp/Rmtlp and its in vivo function. Cofactor binding and substrate interactions. J. Biol. Chem. 275: 31283136.

Milner, C.M. and Campbell, R.D. 1993. The G9a gene in the human major histocompatibility complex encodes a novel protein containing ankyrin-like repeats. Biochem. $I$. 290: $811-818$.

Mowen, K.A., Tang, J., Zhu, W., Schurter, B.T., Shuai, K., Herschman, H.R., and David, M. 2001. Arginine methylation of STAT1 modulates IFNalpha/beta-induced transcription. Cell 104: 731-741.

Murray, K. 1964. The occurrence of e-N-methyllysine in histones. Biochemistry 3: 10-15.

Nakayama, J., Klar, A.J., and Grewal, S.I. 2000. A chromodomain protein, Swi6, performs imprinting functions in fission yeast during mitosis and meiosis. Cell 101: 307-317.

Nakayama, J., Rice, J.C., Strahl, B.D., Allis, C.D., and Grewal, S.I. 2001. Role of histone H3 lysine 9 methylation in epigenetic control of heterochromatin assembly. Science 292: $110-113$.

Nielsen, A.L., Oulad-Abdelghani, M., Ortiz, J.A., Remboutsika, E., Chambon, P., and Losson, R. 2001. Heterochromatin formation in mammalian cells: Interaction between histones and HP1 proteins. Mol. Cell 7: 729-739.

Nielsen, S.J., Schneider, R., Bauer, U.-M., Bannister, A.J., Morrison, A., O'Carroll, D., Firestein, R., Cleary, M., Jenuwein, T., Herreara, R.E., et al. 2001. Rb targets histone H3 methylation and HP1 to promoters. Nature 412: 561-565.

Noma, K., Allis, C.D., and Grewal, S.I. 2001. Transitions in distinct histone $\mathrm{H} 3$ methylation patterns at the heterochromatin domain boundaries. Science 293: 1150-1155.

O'Carroll, D., Scherthan, H., Peters, A.H., Opravil, S., Haynes, A.R., Laible, G., Rea, S., Schmid, M., Lebersorger, A., Jerratsch, M., et al. 2000. Isolation and characterization of Suv39h2, a second histone H3 methyltransferase gene that displays testis-specific expression. Mol. Cell. Biol. 20: 9423 9433.

Pawlak, M.R., Scherer, C.A., Chen, J., Roshon, M.J., and Ruley, H.E. 2000. Arginine N-methyltransferase 1 is required for early postimplantation mouse development, but cells deficient in the enzyme are viable. Mol. Cell. Biol. 20: 48594869.

Pazin, M.J. and Kadonaga, J.T. 1997. What's up and down with histone deacetylation and transcription? Cell 89: 325-328.

Platero, J.S., Hartnett, T., and Eissenberg, J.C. 1995. Functional analysis of the chromodomain of HP1. EMBO J. 14:39773986.

Pollack, B.P., Kotenko, S.V., He, W., Izotova, L.S., Barnoski,
B.L., and Pestka, S. 1999. The human homologue of the yeast proteins Skb1 and Hs17p interacts with Jak kinases and contains protein methyltransferase activity. J. Biol. Chem. 274: 31531-31542.

Rea, S., Eisenhaber, F., O'Carroll, D., Strahl, B.D., Sun, Z.W., Schmid, M., Opravil, S., Mechtler, K., Ponting, C.P., Allis, C.D., et al. 2000. Regulation of chromatin structure by sitespecific histone H3 methyltransferases. Nature 406: 593 599.

Rho, J., Choi, S., Seong, Y.R., Cho, W.K., Kim, S.H., and Im, D.S 2001. Prmt5, which forms distinct homo-oligomers, is a member of the protein-arginine methyltransferase family. J. Biol. Chem. 276: 11393-11401.

Roth, S.Y., Denu, J.M., and Allis, C.D. 2001. Histone acetyltransferases. Annu. Rev. Biochem. 70: 81-120.

Russell, I.D. and Tollervey, D. 1992. NOP3 is an essential yeast protein which is required for pre-rRNA processing. I. Cell Biol. 119: 737-747.

Sassone-Corsi, P., Mizzen, C.A., Cheung, P., Crosio, C., Monaco, L., Jacquot, S., Hanauer, A., and Allis, C.D. 1999. Requirement of Rsk-2 for epidermal growth factor-activated phosphorylation of histone H3. Science 285: 886-891.

Schindler, C. and Darnell, Jr., J.E. 1995. Transcriptional responses to polypeptide ligands: The JAK-STAT pathway. Annu. Rev. Biochem. 64: 621-651.

Schurter, B.T., Koh, S.S., Chen, D., Bunick, G.J., Harp, J.M., Hanson, B.L., Henschen-Edman, A., Mackay, D.R., Stallcup, M.R., and Aswad, D.W. 2001. Methylation of histone h3 by coactivator-associated arginine methyltransferase 1. Biochemistry 40: 5747-5756.

Scott, H.S., Antonarakis, S.E., Lalioti, M.D., Rossier, C., Silver, P.A., and Henry, M.F. 1998. Identification and characterization of two putative human arginine methyltransferases (HRMT1L1 and HRMT1L2). Genomics 48: 330-340.

Shen, E.C., Henry, M.F., Weiss, V.H., Valentini, S.R., Silver, P.A., and Lee, M.S. 1998. Arginine methylation facilitates the nuclear export of hnRNP proteins. Genes \& Dev. 12: 679-691.

Shepherd, G.R., Hardin, J.M., and Noland, B.J. 1971. Methylation of lysine residues of histone fractions in synchronized mammalian cells. Arch. Biochem. Biophys. 143: 1-5.

Stassen, M.J., Bailey, D., Nelson, S., Chinwalla, V., and Harte, P.J. 1995. The Drosophila trithorax proteins contain a novel variant of the nuclear receptor type DNA binding domain and an ancient conserved motif found in other chromosomal proteins. Mech. Dev. 52: 209-223.

Sterner, D.E. and Berger, S.L. 2000. Acetylation of histones and transcription-related factors. Microbiol. Mol. Biol. Rev. 64: 435-459.

Strahl, B.D. and Allis, C.D. 2000. The language of covalent histone modifications. Nature 403: 41-45.

Strahl, B.D., Ohba, R., Cook, R.G., and Allis, C.D. 1999. Methylation of histone $\mathrm{H} 3$ at lysine 4 is highly conserved and correlates with transcriptionally active nuclei in Tetrahymena. Proc. Natl. Acad. Sci. 96: 14967-14972.

Strahl, B.D., Briggs, S.D., Brame, C.J., Caldwell, J.A., Koh, S.S., Ma, H., Cook, R.G., Shabanowitz, J., Hunt, D.F., Stallcup, M.R., and Allis, C.D. 2001. Methylation of histone H4 at arginine 3 occurs in vivo and is mediated by the nuclear receptor coactivator PRMT1. Curr. Biol. 11: 1-5.

Tachibana, M., Sugimoto, K., Fukushima, T., and Shinkai, Y. 2001. SET-domain containing protein, G9a, is a novel lysinepreferring mammalian histone methylthansferase with hyperactivity and specific selectivity to lysines 9 and 27 of histone H3. J. Biol. Chem. 20: 20. 
Tang, J., Gary, J.D., Clarke, S., and Herschman, H.R. 1998. PRMT 3, a type I protein arginine $\mathrm{N}$-methyltransferase that differs from PRMT1 in its oligomerization, subcellular localization, substrate specificity, and regulation. J. Biol. Chem. 273: 16935-16945.

Tang, J., Kao, P.N., and Herschman, H.R. 2000. Protein-arginine methyltransferase I, the predominant protein-arginine methyltransferase in cells, interacts with and is regulated by interleukin enhancer-binding factor 3. I. Biol. Chem. 275: 19866-19876.

Tschiersch, B., Hofmann, A., Krauss, V., Dorn, R., Korge, G., and Reuter, G. 1994. The protein encoded by the Drosophila position-effect variegation suppressor gene Su(var)3-9 combines domains of antagonistic regulators of homeotic gene complexes. EMBO J. 13: 3822-3831.

Turner, B.M. 2000. Histone acetylation and an epigenetic code. BioEssays 22: 836-845.

van Holde, K.E. 1988. Histone modifications. In Chromatin, Springer series in molecular biology (ed. A. Rich), pp. 111148. Springer, New York.

Vandel, L. and Trouche, D. 2001. Physical association between the histone acetyl transferase CBP and a histone methyl transferase. EMBO Rep. 2: 21-26.

Vignali, M., Hassan, A.H., Neely, K.E., and Workman, J.L. 2000. ATP-dependent chromatin-remodeling complexes. Mol. Cell. Biol. 20: 1899-1910.

Wang, H., Huang, Z.Q., Xia, L., Feng, Q., Erdjument-Bromage, H., Strahl, B.D., Briggs, S.D., Allis, C.D., Wong, J., Tempst, P., et al. 2001. Methylation of histone $\mathrm{H} 4$ at arginine $3 \mathrm{fa}-$ cilitates transcriptional activation by nuclear hormone receptor. Science 31: 31.

Wang, L., Liu, L., and Berger, S.L. 1998. Critical residues for histone acetylation by Gen5, functioning in Ada and SAGA complexes, are also required for transcriptional function in vivo. Genes \& Dev. 12: 640-653.

Wei, Y., Yu, L., Bowen, J., Gorovsky, M.A., and Allis, C.D. 1999. Phosphorylation of histone $\mathrm{H} 3$ is required for proper chromosome condensation and segregation. Cell 97: 99-109.

Weiss, V.H., McBride, A.E., Soriano, M.A., Filman, D.J., Silver, P.A., and Hogle, J.M. 2000. The structure and oligomerization of the yeast arginine methyltransferase, Hmt1. Nature Struct. Biol. 7: 1165-1171.

Wu, J. and Grunstein, M. 2000. 25 years after the nucleosome model: Chromatin modifications. Trends Biochem. Sci. 25: 619-623.

Zhang, X., Zhou, L., and Cheng, X. 2000. Crystal structure of the conserved core of protein arginine methyltransferase PRMT3. EMBO J. 19: 3509-3519.

Zheng, Q., Simel, E.J., Klein, P.E., Royer, M.T., and Houtz, R.L. 1998. Expression, purification, and characterization of recombinant ribulose-1,5-bisphosphate carboxylase/oxygenase large subunit ne-methyltransferase. Protein Expr. Purif. 14: 104-112. 


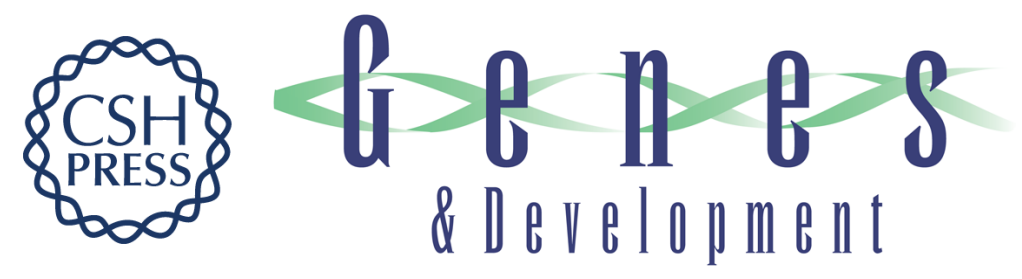

\section{Transcription regulation by histone methylation: interplay between different covalent modifications of the core histone tails}

Yi Zhang and Danny Reinberg

Genes Dev. 2001, 15:

Access the most recent version at doi:10.1101/gad.927301

References This article cites 102 articles, 46 of which can be accessed free at: http://genesdev.cshlp.org/content/15/18/2343.full.html\#ref-list-1

License

Email Alerting

Receive free email alerts when new articles cite this article - sign up in the box at the top Service right corner of the article or click here.

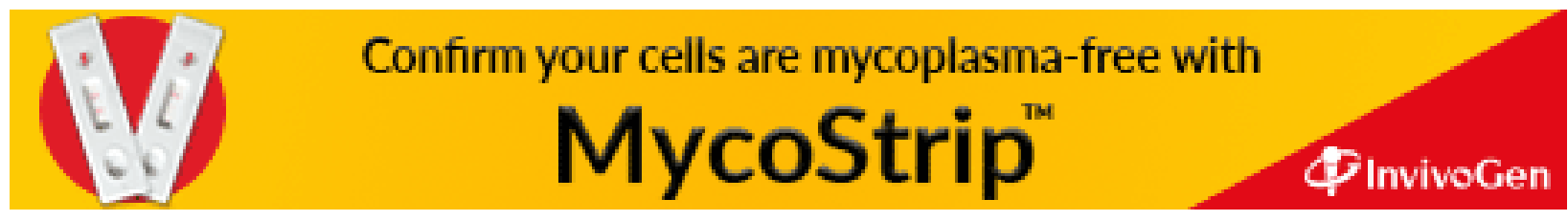

